\title{
Tumor mutational burden in lung cancer: a systematic literature review
}

\author{
Connor Willis ${ }^{1}$, Michelle Fiander ${ }^{1}$, Dao Tran², Beata Korytowsky ${ }^{3}$, John-Michael \\ Thomas $^{3}$, Florencio Calderon ${ }^{3}$, Teresa M. Zyczynski ${ }^{3}$, Diana Brixner ${ }^{1}$, David D. \\ Stenehjem ${ }^{1,2}$ \\ ${ }^{1}$ Department of Pharmacotherapy, University of Utah, Salt Lake City, UT, USA \\ ${ }^{2}$ Department of Pharmacy Practice and Pharmaceutical Sciences, University of Minnesota, Duluth, MN, USA \\ ${ }^{3}$ Bristol-Myers Squibb Company, Lawrenceville, NJ, USA \\ Correspondence: David Stenehjem, email: stene032@d.umn.edu \\ Keywords: biomarkers, chemotherapy, immunotherapy, lung cancer, PD-L1 \\ Received: June 14, $2019 \quad$ Accepted: September 16, $2019 \quad$ Published: November 12, 2019 \\ Copyright: Willis et al. This is an open-access article distributed under the terms of the Creative Commons Attribution License 3.0 \\ (CC BY 3.0), which permits unrestricted use, distribution, and reproduction in any medium, provided the original author and source \\ are credited.
}

\section{ABSTRACT}

Purpose: To assess the association of tumor mutational burden (TMB) with clinical outcomes, other biomarkers and patient/disease characteristics in patients receiving therapy for lung cancer.

Results: In total, 4,303 publications were identified; 81 publications were included. The majority of publications assessing clinical efficacy of immunotherapy reported an association with high TMB, particularly when assessing progression-free survival and objective response rate. High TMB was consistently associated with TP53 alterations, and negatively associated with EGFR mutations. High TMB was also associated with smoking, squamous cell non-small cell lung carcinoma, and being male.

Methods: A systematic literature review based upon an a priori protocol was conducted following Preferred Reporting Items for Systematic Reviews and MetaAnalyses (PRISMA) and Cochrane methodologies. Searches were conducted in EMBASE, SCOPUS, Ovid MEDLINE ${ }^{\circledR}$, and Emcare (from January 2012 until April 2018) and in two clinical trial registries. Conference abstracts were identified in EMBASE, and in targeted searches of recent major conference proceedings (from January 2016 until April 2018). Publications reporting data in patients receiving therapy for lung cancer that reported TMB and its association with clinical efficacy, or with other biomarkers or patient/disease characteristics, were included. Results are presented descriptively.

Conclusion: This systematic literature review identified several clinical outcomes, biomarkers, and patient/disease characteristics associated with high TMB, and highlights the need for standardized definitions and testing practices. Further studies using standardized methodology are required to inform treatment decisions.

\section{INTRODUCTION}

The advent of immunotherapy has transformed the clinical oncology landscape in recent years, with significant improvements in long-term survival in some patients. However, a large proportion of patients do not respond to immunotherapies, and predictors of response are required to improve patient selection. Among investigated biomarkers, tumor mutational burden (TMB) has recently emerged as a potential predictor of response to immunotherapy in various tumor types $[1,2]$. Increases in TMB are driven by several factors, including 
DNA replication errors mediated by defective tumor suppressor genes (e. g. TP53), deficient DNA mismatch repair (dMMR) mechanisms (generally indicated by high microsatellite instability [MSI-H]), and exposure to mutagens such as tobacco, alkylating agents and ultraviolet light $[3,4]$. Tumors with high levels of TMB are thought to express more cancer-specific antigens (neoantigens) that may sensitize them to immunotherapy [1, 5-7]. Accordingly, TMB levels have been shown to correlate with objective response rates (ORR) during immunotherapy across a number of cancer types [8].

Immunohistochemistry-determined programmed death ligand 1 (PD-L1) expression has been approved by the US Food and Drug Administration (FDA) as a companion diagnostic for several immunotherapies in various cancers [9]. In March 2015, the programmed cell death-1 (PD-1) inhibitor nivolumab was approved for second-line therapy of metastatic NSCLC independent of PD-L1 expression, as therapeutic response in phase 3 programs was largely independent of PD-L1 levels [10-12]. Conversely, in October 2015, the PD-1 inhibitor pembrolizumab received PD-L1-dependent FDA approval for second-line therapy of metastatic non-small cell lung cancer (NSCLC), based on observations that improved efficacy was associated with elevated PD-L1 expression [13]. Collectively, these studies reported that many patients with elevated PD-L1 levels did not respond to immunotherapy, while a substantial minority of patients who had low PD-L1 expression did experience clinical benefit [10-13]. These findings highlight the need for additional biomarkers to improve patient selection for these therapies.

Tumors with dMMR/MSI-H have demonstrated improved response rates to PD-1/PD-L1 inhibitors, and $\mathrm{dMMR} / \mathrm{MSI}-\mathrm{H}$ deficiency has become the first "tissueagnostic" biomarker to receive FDA approval, for therapy with pembrolizumab [14]. However, the vast majority of tumor samples with high TMB do not exhibit dMMR/ MSI-H [4], and non-dMMR/MSI-H deficient tumors may benefit from immunotherapy. Lung cancers are more frequently associated with high TMB compared with other cancer types [4, 15], and studies suggest an association between TMB and response to immunotherapy in patients with NSCLC $[10,16,17]$.

Traditional evaluation of tissue TMB by whole exome sequencing (WES) using next-generation sequencing (NGS) technology has been expensive and labor-intensive [18]. Recent advances in comprehensive genomic profiling (CGP), using targeted NGS, which measures the number of mutations on a portion of the coding region while simultaneously providing data on specific DNA alterations, have been validated [19] and shown to reflect measurements obtained by WES [20]. Based on parallel FDA approval, the Centers for Medicare \& Medicaid Services proposed coverage for the FoundationOne CDx in vitro diagnostic test for
NGS evaluation of gene mutations in solid tumors [21]. These developments have made routine TMB evaluation increasingly feasible. Nevertheless, the methods of reporting TMB in lung cancer remain highly inconsistent; some studies report TMB in terms of the absolute number of mutations, while others assess mutations per DNA megabase (mut/Mb). Additionally, thresholds used to denote high TMB vary greatly and no widely used standard currently exists.

This is the first systematic literature review describing the role of TMB as a predictive biomarker in patients with lung cancer. We aimed to assess associations between TMB and clinical efficacy outcomes in patients receiving therapy for lung cancer, to identify other biomarkers related to $\mathrm{TMB}$, and to understand the association of patient and disease characteristics with TMB. Additionally, we sought to describe how TMB testing is implemented in clinical practice and reported in the literature.

\section{RESULTS}

\section{Publication screening}

Searches retrieved 4,303 publications in total, of which 1,298 were duplicates, 2,201 were excluded based on titles and abstracts, and 723 were excluded based on full-text; 81 studies were included (Figure 1). Most articles identified were published in 2017 (54 publications; $65.9 \%$ ), with 16 (19.5\%) published from January to April 2018. A summary of relevant data reported by publications presenting efficacy outcomes, biomarkers and/or patient or disease characteristics is presented in Supplementary Table 4.

\section{Clinical efficacy outcomes and TMB}

In total, 22 publications presenting data on TMB also reported one or more clinical outcomes (Table 1) [1, $10,16,27-45]$. The results of our risk of bias analysis are summarized in Supplementary Figure 1. Of these, one publication reported on patients with small cell lung cancer (SCLC) [32], one reported on a combination of NSCLC and SCLC [36], and the rest reported on patients with NSCLC. Of the 22 publications that reported clinical outcomes, 14 used CGP to assess TMB [16, 28, 30, 33, 34, 36-41, 43-45] while eight studies used WES $[1,10,27,29,31,32,35,42]$. Of the 13 publications assessing TMB using CGP, six used a Foundation Medicine platform [16, 28, 30, 33, 41, 45], three used a combination of commercial platforms (including Foundation Medicine, Guardant360, Caris Life Sciences, and Precipio) [37, 40, 44], one used only Caris platform results [38], one used only MSK-IMPACT results [34], and three did not specify which testing platforms were used $[36,39,43]$. 


\section{Overall survival}

Twelve publications presented data on overall survival (OS) and TMB (Table 2) [10, 16, 27-30, 32, $37,38,41,43,44]$. Of these, ten publications assessed patients receiving immunotherapies $[10,16,28-30$, $32,37,38,41,44]$, with two assessing more than one treatment arm [30,32]. Eight of these (including the one study assessing SCLC) reported longer OS in patients with high TMB compared with low TMB [10, 16, 28, $30,32,37,41,44]$; yet only two reported statistical significance $[28,41]$. Two publications reported a nonsignificant association between TMB and OS during immunotherapy, but provided no details on the definition of high TMB and did not present median OS [29, 38]. Two further publications presented data for OS and TMB but did not specify the type of treatment used [27, 43]; one of these reported that OS was significantly longer in patients with low TMB compared with high TMB [43], with the other publication reporting no significant difference [27].

\section{Progression-free survival}

Eleven publications presented data for progressionfree survival (PFS) and TMB (Table 2) [1, 10, 16, 27,
$30,32-34,36,39,42]$. Of these, nine publications (including the one SCLC study) assessed patients receiving immunotherapies $[1,10,16,30,32-34,36$, 39], with all reporting longer PFS in patients with high TMB compared with low TMB. Six of these publications also indicated that the difference in PFS was significant, either by hazard ratio confidence intervals (CIs) less than 1 , or by $P$ value $[1,16,30,34,36,39]$. Two publications presented data for PFS and TMB in patients receiving chemotherapy, with equivocal differences in PFS and no statistical comparison presented [10,33]. Two further publications did not specify the type of treatment received $[27,42]$; one of these reported that PFS was significantly shorter in patients with high TMB compared with low TMB [42], with the other reporting no significant difference [27].

\section{Objective response rate}

Eight publications presented data for ORR and TMB in patients receiving immunotherapies (including the one SCLC study) (Table 2) [1, 10, 16, 29-32, 39]; all reported higher ORR in patients with high TMB compared with low TMB. Three of these publications reported statistically significant differences $[1,29,31]$, with

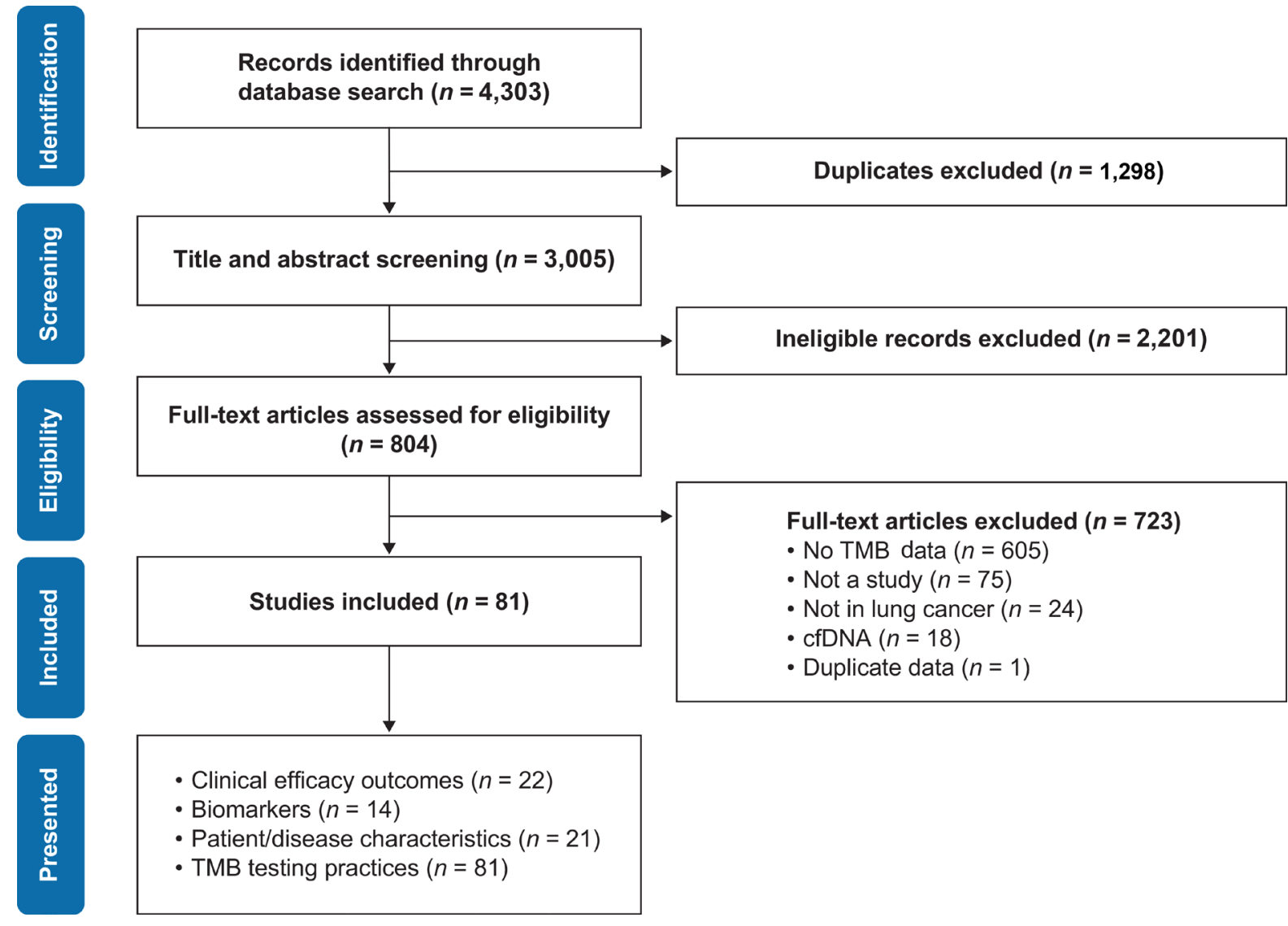

Figure 1: PRISMA flow diagram of publication identification process. TMB, tumor mutational burden. 'Presented' categories are not mutually exclusive. 
Table 1: Summary of characteristics of publications reporting efficacy data

\begin{tabular}{|c|c|c|c|c|}
\hline Publication & $\begin{array}{l}\text { Lung cancer } \\
\text { type }\end{array}$ & TMB threshold values & Agent/Setting & $\begin{array}{c}\text { TMB-H patients/total } \\
\text { patients assessed for } \\
\text { TMB }(n / N)\end{array}$ \\
\hline $\begin{array}{l}\text { Carbone } 2017 \\
{[10]}\end{array}$ & NSCLC & $\begin{array}{l}\text { TMB-L: }<100 \text { mutations } \\
\text { TMB-I: } 100-242 \text { mutations } \\
\text { TMB-H: }>242 \text { mutations }\end{array}$ & $\begin{array}{l}\text { Nivolumab/1L } \\
\text { Platinum-based } \\
\text { chemotherapy }\end{array}$ & $\begin{array}{l}47 / 158 \\
60 / 154\end{array}$ \\
\hline Choi 2017 [27] & NSCLC & NR & NR & NR/108 \\
\hline Davis 2017 [28] & NSCLC & $\begin{array}{l}\text { TMB-L: }<15 \text { mut/Mb } \\
\text { TMB-H: } \geq 15 \text { mut/Mb }\end{array}$ & PD-1/PD-L1 inhibitors & $\mathrm{NR} / 35$ \\
\hline $\begin{array}{l}\text { Gettinger } 2017 \\
{[29]}\end{array}$ & NSCLC & NR & PD-1/PD-L1 inhibitors & $\mathrm{NR} / 45$ \\
\hline $\begin{array}{l}\text { Goodman } 2017 \\
{[16]}\end{array}$ & NSCLC & $\begin{array}{l}\text { TMB-L: } 1-5 \text { mut/Mb } \\
\text { TMB-I: 6-19 mut/Mb } \\
\text { TMB-H: } \geq 20 \text { mut/Mb }\end{array}$ & PD-1/PD-L1 inhibitors & $3 / 36$ \\
\hline $\begin{array}{l}\text { Haratani } 2017 \\
{[31]}\end{array}$ & NSCLC & NR & Nivolumab & $\mathrm{NR} / 9$ \\
\hline $\begin{array}{l}\text { Hellmann } 2017 \\
\text { [34] }\end{array}$ & NSCLC & $\begin{array}{l}\text { TMB-L: Below } 85 \text { th percentile } \\
\text { TMB-H: Above } 85 \text { th percentile }\end{array}$ & $\begin{array}{l}\text { PD-L1 inhibitors +/- anti- } \\
\text { CTLA-4 therapy }\end{array}$ & $\mathrm{NR} / 437$ \\
\hline $\begin{array}{l}\text { Hellmann } 2018 \\
\text { [33] }\end{array}$ & NSCLC & $\begin{array}{l}\text { TMB-L: }<10 \mathrm{mut} / \mathrm{Mb} \\
\text { TMB-H: } \geq 10 \mathrm{mut} / \mathrm{Mb}\end{array}$ & $\begin{array}{l}\text { Nivolumab + ipilimumab } \\
\text { Chemotherapy }\end{array}$ & $\begin{array}{l}139 / 330 \\
160 / 349\end{array}$ \\
\hline $\begin{array}{l}\text { Hellmann } 2018 \\
\text { [32] }\end{array}$ & SCLC & $\begin{array}{l}\text { TMB-L: < } 143 \text { mutations } \\
\text { TMB-I: } 143-247 \text { mutations } \\
\text { TMB-H: }>247 \text { mutations }\end{array}$ & $\begin{array}{l}\text { Nivolumab } \\
\text { Nivolumab + ipilimumab }\end{array}$ & $\begin{array}{c}47 / 133 \\
26 / 78\end{array}$ \\
\hline Hu 2018 [35] & NSCLC & TMB-H: $\geq 20$ mut/Mb & PD-1/PD-L1 inhibitors & 9/NR \\
\hline $\begin{array}{l}\text { Kowanetz } 2017 \\
{[30]}\end{array}$ & NSCLC & $\begin{array}{l}\text { TMB-L: Below 50th percentile } \\
\text { TMB-H: Above } 50 \text { th percentile }\end{array}$ & $\begin{array}{l}\text { Atezolizumab/1L } \\
\text { Atezolizumab/2L }\end{array}$ & $\begin{array}{l}\mathrm{NR} / 102 \\
\mathrm{NR} / 371\end{array}$ \\
\hline $\begin{array}{l}\text { Mahadevan } \\
2017[36]\end{array}$ & $\begin{array}{c}\text { NSCLC } \\
(n=80) \text { and } \\
\text { SCLC }(n=5)\end{array}$ & $\begin{array}{l}\text { TMB-L: Below 50th percentile } \\
\text { TMB-H: Above 50th percentile }\end{array}$ & $\begin{array}{l}\text { PD-1 }(n=82) / \\
\text { PD-L1 }(n=5) \text { inhibitors/ } \\
\text { other agents }(n=7)\end{array}$ & NR/94 \\
\hline Park 2017 [37] & NSCLC & $\begin{array}{l}\text { TMB-L: } 1-5 \text { mut/Mb } \\
\text { TMB-I: 6-19 mut/Mb } \\
\text { TMB-H: > } 20 \text { mut/Mb }\end{array}$ & Nivolumab & $\mathrm{NR} / 36$ \\
\hline Patel 2017 [38] & NSCLC & NR & Immunotherapy & $\mathrm{NR} / 50$ \\
\hline Rizvi 2015 [1] & NSCLC & $\begin{array}{l}\text { TMB-L: Below } 50 \text { th percentile } \\
\text { TMB-H: Above } 50 \text { th percentile }\end{array}$ & $\begin{array}{l}\text { Pembrolizumab (cohort 1) } \\
\text { Pembrolizumab (cohort 2) }\end{array}$ & $\begin{array}{l}\mathrm{NR} / 16 \\
\mathrm{NR} / 18\end{array}$ \\
\hline Ross 2017 [45] & NSCLC & NR & $\begin{array}{l}\text { Immune checkpoint } \\
\text { inhibitors }\end{array}$ & $545 / 3758$ \\
\hline $\begin{array}{l}\text { Roszik } 2016 \\
\text { [39] }\end{array}$ & NSCLC & $\begin{array}{l}\text { TMB-L: }<100 \text { mutations } \\
\text { TMB-H: } \geq 100 \text { mutations }\end{array}$ & Pembrolizumab & $21 / 29$ \\
\hline $\begin{array}{l}\text { Rozenblum } \\
2017 \text { [40] }\end{array}$ & NSCLC & NR & $\begin{array}{l}\text { Pembrolizumab and } \\
\text { nivolumab }\end{array}$ & $\mathrm{NR} / 18$ \\
\hline
\end{tabular}




\begin{tabular}{|c|c|c|c|c|}
\hline $\begin{array}{l}\text { Singal } 2017 \\
{[41]}\end{array}$ & NSCLC & $\begin{array}{l}\text { TMB-L: } 1-5 \mathrm{mut} / \mathrm{Mb} \\
\text { TMB-I: } 6-19 \mathrm{mut} / \mathrm{Mb} \\
\text { TMB-H: } \geq 20 \mathrm{mut} / \mathrm{Mb}\end{array}$ & Nivolumab & $\mathrm{NR} / 444$ \\
\hline Wang 2017 [42] & NSCLC & $\begin{array}{l}\text { TMB-L: Below 50th percentile } \\
\text { TMB-H: Above 50th percentile }\end{array}$ & NR & $\mathrm{NR} / 98$ \\
\hline Xiao 2016 [43] & NSCLC & $\begin{array}{l}\text { TMB-L: } \leq 4 \text { mutations } \\
\text { TMB-H: }>4 \text { mutations }\end{array}$ & NR & $47 / 335$ \\
\hline $\begin{array}{l}\text { Yaghmour } 2016 \\
{[44]}\end{array}$ & NSCLC & $\begin{array}{l}\text { TMB-L: Below 80th percentile } \\
\text { TMB-H: Above 80th percentile }\end{array}$ & $\begin{array}{l}\text { Nivolumab, } \\
\text { pembrolizumab, or } \\
\text { ipilimumab }\end{array}$ & $3 / 23$ \\
\hline
\end{tabular}

Abbreviations: 1L, first-line; CTLA-4, cytotoxic T-lymphocyte-associated protein 4; mut/Mb, mutations per DNA megabase; NR, not reported; NSCLC, non-small cell lung cancer; PD-1, programmed cell death-1; PD-L1, programmed death ligand 1; SCLC, small cell lung cancer; TMB, tumor mutational burden; TMB-H, high TMB; TMB-I, intermediate TMB; TMB-L, low TMB.

another reporting no significant difference [16]. The other four publications did not present statistical comparisons. One publication also presented data for ORR and TMB in patients receiving platinum-based chemotherapy; there appeared to be no association of TMB on ORR in these patients [10].

\section{Durable clinical benefit}

Four publications presented data on durable clinical benefit (DCB) and TMB in patients receiving immunotherapies (Supplementary Table 5) [1, 34, 36, 39]. Three reported that TMB was significantly higher in patients who experienced DCB compared with those who did not $[1,34,36]$. One further publication reported patients with high TMB were more likely to experience DCB than patients with low TMB, but did not present statistical comparisons [39].

\section{Disease control}

Two studies presented data on disease control rate (DCR) and TMB in patients receiving immunotherapy (Supplementary Table 5) [35, 41]. One of these reported that DCR was significantly higher in patients with high TMB compared with patients with low TMB [41]. The other publication included only patients with high TMB, but reported DCR of $100 \%$ [35].

An additional publication reported no clear association of TMB levels with response as determined by Response Evaluation Criteria In Solid Tumors (RECIST) criteria (partial response, stable disease, or progressive disease) in patients receiving immunotherapies (Supplementary Table 5) [40].

\section{Duration of treatment}

Two publications compared duration of therapy (DoT) with immunotherapy in patients with high and low TMB; both of these reported that DoT was significantly longer in patients with high TMB (Supplementary Table 5) [41, 45].

\section{Biomarkers and TMB}

A total of 14 publications reporting the association of TMB with biomarkers were identified (Table 3) [10, $27,36,45-55]$. Nine publications reported data on PD-L1 and TMB [10, 27, 36, 45, 48-50, 53, 55]. Four reported significant associations of PD-L1 and TMB [45, 48, 53, 55], with two reporting weak Spearman's correlations of 0.12 and $0.085[45,48]$. Three publications (one of which presented three analyses from different centers) assessed TMB and EGFR status; all reported that EGFR-mut was significantly associated with low TMB [46, 47, 51]. All three publications assessing TP53 alterations reported that it was significantly associated with high TMB $[51,52,54]$.

\section{Patient/disease characteristics and TMB}

We identified 22 publications that evaluated the association between patient or disease characteristics and TMB (Table 4). Smoking history was the most commonly reported; of the 16 publications presenting data on smoking history and TMB [1, 28, 36, 42, 43, 51, 56-65], 14 reported that smoking was significantly associated with higher TMB levels $[1,36,42,43,51,57,59-62$, $64,65]$ or with a greater likelihood of a patient having high TMB $[28,56]$. Two further publications assessed TMB levels in patients with a history of smoking; one did not assess significance [63], and the other reported nonsignificance [58].

Five publications presented data on TMB in NSCLC stratified by squamous or adenocarcinoma histology (Table 4) [51, 54, 60, 66, 67]. All reported higher TMB in patients with squamous cell or 
Table 2: Publications reporting primary efficacy outcomes (OS, PFS, ORR) in patients receiving active therapy for lung cancer

\begin{tabular}{|c|c|c|c|}
\hline & $\begin{array}{l}\text { Median OS (Months) } \\
{[\text { HR }(95 \% \text { CI }) / P \text { Value] }}\end{array}$ & $\begin{array}{l}\text { Median PFS (Months) } \\
{[\text { HR }(95 \% \text { CI) } / P \text { Value] }}\end{array}$ & $\begin{array}{l}\text { ORR }(\%) \\
{[P \text { Value }]}\end{array}$ \\
\hline \multicolumn{4}{|l|}{ IMMUNOTHERAPY } \\
\hline \multicolumn{4}{|l|}{ Carbone 2017 [10] } \\
\hline Nivolumab/1L & $\begin{array}{l}\text { Low/intermediate vs high } \\
\text { TMB: } 12.7 \text { vs } 18.3[P: \mathrm{NR}]\end{array}$ & $\begin{array}{l}\text { Low vs intermediate vs high } \\
\text { TMB: } 6.9 \text { vs } 6.5 \text { vs } 9.7 \\
{[P: \text { NR }]}\end{array}$ & $\begin{array}{l}\text { Low/intermediate vs high TMB: } \\
23 \% \text { vs } 47 \%[P: \mathrm{NR}]\end{array}$ \\
\hline \multicolumn{4}{|l|}{ Davis 2017 [28] } \\
\hline PD-1/PD-L1 inhibitors & $\begin{array}{l}\text { Longer with high TMB } \\
{[0.19(0.04 \text { to } 0.88) ; P=.034]}\end{array}$ & NR & NR \\
\hline \multicolumn{4}{|l|}{ Gettinger 2017 [29] } \\
\hline PD-1/PD-L1 inhibitors & $\mathrm{NR}[P=.92]$ & NR & Higher with high TMB $[P=.02]$ \\
\hline \multicolumn{4}{|l|}{ Goodman 2017 [16] } \\
\hline PD-1/PD-L1 inhibitors & $\begin{array}{l}\text { Low/intermediate vs high } \\
\text { TMB: } 7.6 \text { vs not reached } \\
{[0.32(0.07 \text { to } 1.50) ; P=.15]}\end{array}$ & $\begin{array}{l}\text { Low/intermediate vs high } \\
\text { TMB: } 2.1 \text { vs } 12.5[0.32 \\
\text { (0.13 to } 0.81) ; P=.0817]\end{array}$ & $\begin{array}{l}\text { Low/intermediate vs high TMB: } \\
18 \% \text { vs } 33 \% \\
{[P=.4882]}\end{array}$ \\
\hline
\end{tabular}

Kowanetz 2017 [30]

Atezolizumab/1L

Longer with high TMB [50th Longer with high TMB percentile: 0.79 (0.39 to 1.58$), \quad$ [50th percentile: 0.58

(0.36 to 0.94$), P: \mathrm{NR} ; 75$ th

Low vs high TMB (50th percentile): $P$ : NR; 75th percentile: 0.45

(0.17 to 1.16$), P: \mathrm{NR}]$ percentile: 0.54 (0.3 to 0.97$)$, $P: \mathrm{NR}]$ $13 \%$ vs $28 \%$ [ $P$ : NR]

Low vs high TMB

(75th percentile): $20 \%$ vs $25 \%$ [P: NR]

Atezolizumab/2L

Longer with high TMB

Longer with high TMB [50th percentile: 0.87 ( 0.65 to

[50th percentile: 0.64 ( 0.5 to

Low vs high TMB (50th percentile): 1.16), $P$ : NR; 75th percentile: $\quad 0.8), P$ : NR; 75th percentile: $14 \%$ vs $25 \%[P:$ NR] $0.7(0.49$ to 1.0$), P: \mathrm{NR}] \quad 0.5(0.38$ to 0.67$), P: \mathrm{NR}]$

Low vs high TMB (75th percentile): $16 \%$ vs $29 \%$ [P: NR]

\begin{tabular}{|c|c|c|c|}
\hline \multicolumn{4}{|l|}{ Haratani 2017 [31] } \\
\hline Nivolumab & NR & NR & Higher with high TMB $[P=.038]$ \\
\hline \multicolumn{4}{|l|}{ Hellmann 2017 [34] } \\
\hline $\begin{array}{l}\text { Anti-PD-L1 +/- anti-CTLA-4 } \\
\text { therapy }\end{array}$ & NR & $\begin{array}{l}\text { Longer with high TMB }[\mathrm{HR}: \\
0.59(95 \% \mathrm{CI}: \mathrm{NR}) ; P=.004]\end{array}$ & NR \\
\hline \multicolumn{4}{|l|}{ Hellmann 2018 [33] } \\
\hline Nivolumab + Ipilimumab & NR & $\begin{array}{l}\text { Low vs high TMB: } 3.2 \text { vs } 7.2 \\
{[P: \mathrm{NR}]}\end{array}$ & NR \\
\hline
\end{tabular}

Hellmann 2018 [32]

Nivolumab

Low/intermediate vs high

Low/intermediate vs high TMB: 3.1 vs 5.4 [P: NR]

TMB: 1.3 vs 1.4 [P: NR]

Low/intermediate vs high TMB: 7\% vs $21 \%$ [P: NR]

Nivolumab + ipilimumab Intermediate vs high TMB: 3.4 Low/intermediate vs high Low/intermediate vs high TMB: vs 22.0 [P: NR] TMB: 1.3 vs $7.8[P: \mathrm{NR}]$

$22 \%$ vs $46 \%$ [ $P$ : NR]

$\begin{array}{ll}\text { Mahadevan } 2017[36] & \\ \text { PD-1/PD-L1 inhibitors } \quad \text { NR } & \text { Longer with high TMB } \quad \text { NR } \\ & {[P=.015]}\end{array}$

Park 2017 [37]

Nivolumab

Low vs intermediate vs high NR

NR

TMB: 12.4 vs 10.3 vs not

reached $[P=.211]$

\section{Patel 2017 [38]}

Immunotherapy

NR $[P=.5]$

NR

NR 


\section{Rizvi 2015 [1]}

$\begin{array}{llll}\text { Pembrolizumab (cohort 1) } & \text { NR } & \text { Low/intermediate vs high } & \text { Low vs high TMB: } 0 \% \text { vs } 63 \% \\ & & \text { TMB: } 3.7 \text { vs } 14.5[P=.01] & {[P=.03]} \\ \text { Pembrolizumab (cohort 2) } & \text { NR } & \text { Low/intermediate vs high } & \text { Low vs high TMB: } 22 \% \text { vs } 56 \% \\ & & \text { TMB: } 3.4 \text { vs not reached } & \text { [NR }] \\ & {[P=.006]} & \end{array}$

\begin{tabular}{|c|c|c|c|}
\hline \multicolumn{4}{|l|}{ Roszik 2016 [39] } \\
\hline Pembrolizumab & NR & $\begin{array}{l}\text { Low vs high TMB: } 4.1 \text { vs } 8.3 \\
{[P=.0003]}\end{array}$ & $\begin{array}{l}\text { Low vs high TMB: } 0 \% \text { vs } 48 \% \\
\text { [NR] }\end{array}$ \\
\hline \multicolumn{4}{|l|}{ Singal 2017 [41] } \\
\hline Nivolumab & $\begin{array}{l}\text { Low/intermediate vs high } \\
\text { TMB: } 10 \text { vs not reached } \\
{[P<.01]}\end{array}$ & NR & NR \\
\hline \multicolumn{4}{|l|}{ Yaghmour 2016 [44] } \\
\hline $\begin{array}{l}\text { Nivolumab, pembrolizumab, } \\
\text { or ipilimumab }\end{array}$ & $\begin{array}{l}\text { Low vs high TMB: } 4.9 \text { vs not } \\
\text { reached [HR undefined ( } 95 \% \\
\text { CI: } 0.04 \text { to } 1.90) ; P=.21 \text { ] }\end{array}$ & NR & NR \\
\hline \multicolumn{4}{|l|}{ CHEMOTHERAPY } \\
\hline \multicolumn{4}{|l|}{ Carbone 2017 [10] } \\
\hline $\begin{array}{l}\text { Platinum-based } \\
\text { chemotherapy }\end{array}$ & NR & $\begin{array}{l}\text { Low vs intermediate vs high } \\
\text { TMB: } 4.2 \text { vs } 3.6 \text { vs } 5.8 \\
{[\mathrm{NR}]}\end{array}$ & $\begin{array}{l}\text { Low/intermediate vs high TMB: } \\
33 \% \text { vs } 28 \% \text { [NR] }\end{array}$ \\
\hline \multicolumn{4}{|l|}{ Hellmann 2018 [33] } \\
\hline Chemotherapy & NR & $\begin{array}{l}\text { Low vs high TMB: } 5.5 \text { vs } 5.5 \\
\text { [NR] }\end{array}$ & NR \\
\hline NOT SPECIFIED & & & \\
\hline
\end{tabular}

Choi 2017 [27]

$\begin{array}{clll}\text { Not specified } & \text { NR }[P=.5933] & \text { NR }[P=.7765] & \text { NR } \\ \text { Wang 2017*[42] } & \text { NR } & \begin{array}{l}\text { Shorter with high } \text { TMB }^{*} \\ {[P=.0133]}\end{array} & \text { NR } \\ \text { Not specified } & & {[P=}\end{array}$

Xiao 2016 [43]

Not specified

Low vs high TMB: 61 vs $48.4 \quad$ NR

NR

$[P=.02]$

Abbreviations: CI, confidence interval; HR, hazard ration; NR, not reported; ORR, objective response rate; OS, overall survival; PFS, progression-free survival; TMB, tumor mutational burden.

${ }^{*}$ Wang 2017 presents disease-free survival, not progression-free survival.

non-adenocarcinoma compared with patients with adenocarcinoma $[51,60,66,67]$; however, only one demonstrated a significant difference [51]. Four publications assessed lung cancer stage, with all reporting no significant association with TMB $[27,43$, $58,65]$.

\section{Demographics}

All three publications assessing TMB and sex reported that men had significantly higher TMB than women (Table 4) [43, 51, 65]. Four studies [42, 43, 60, 65] assessed age and TMB. Three reported higher TMB in older patients $[42,60,65]$, with one demonstrating significance [42]. One publication investigated age in four subgroups across TP53 status and cancer histology [68]. This study reported a small, but significant, inverse association of age and TMB in patients with TP53-mut adenocarcinoma, but no significant effect of age and TMB in patients with TP53-wt adenocarcinoma, or with TP53-wt or TP53-mut squamous cell carcinoma.

\section{TMB testing practices}

A summary of the TMB testing practices is presented in Table 5. Among the 81 publications 
Table 3: Publications reporting TMB and relevant biomarkers

\begin{tabular}{|c|c|c|}
\hline Publication & Main findings relating to TMB and biomarker & $P$ Value \\
\hline \multicolumn{3}{|l|}{ PD-1/PD-L1 } \\
\hline Carbone 2017 [10] & Pearson's r: 0.059 & NA \\
\hline \multirow[t]{3}{*}{ Choi 2017 [27] } & Pearson's r: & \\
\hline & PD-1: 0.004 & PD-1: .96958 \\
\hline & PD-L1: -0.067 & PD-L1: .52563 \\
\hline Goldberg 2017 [48] & Spearman's rho: 0.12 & .00035 \\
\hline Liu 2018 [49] & Spearman's rho: 0.092 & .62 \\
\hline Mahadevan 2017 [36] & Undefined & .47 \\
\hline Nakagomi 2018 [50] & Undefined & .49 \\
\hline Schabath 2017 [53] & Undefined & .03 \\
\hline Senarathne 2018 [55] & Undefined & .05 \\
\hline Ross 2017 [45] & Spearman's rho: 0.085 & .00062 \\
\hline \multicolumn{3}{|l|}{ EGFR } \\
\hline Chen 2017 [46] & Median TMB higher in EGFR-wt patients (8.4) vs EGFR-mut patients (4.6) & .034 \\
\hline \multicolumn{3}{|l|}{ Dong 2017 [47] } \\
\hline TCGA & Median TMB higher in EGFR-wt patients (181) vs EGFR-mut patients (56) & $<.001$ \\
\hline Broad Institute & Median TMB higher in EGFR-wt patients (209) vs EGFR-mut patients (59) & .003 \\
\hline $\begin{array}{l}\text { Guangdong Lung } \\
\text { Cancer Institute }\end{array}$ & Median TMB higher in EGFR-wt patients (197) vs EGFR-mut patients (162) & .029 \\
\hline Owada $2017[51]$ & Median TMB higher in EGFR-wt patients vs EGFR-mut patients (values NR) & $<.001$ \\
\hline \multicolumn{3}{|l|}{ TP53 } \\
\hline Owada $2017[51]$ & $\begin{array}{l}\text { Median TMB higher in TP53-positive patients vs TP53-negative patients } \\
\text { (values NR) }\end{array}$ & $<.001$ \\
\hline Rothberg 2017 [52] & $\begin{array}{l}\text { Median TMB higher in TP53-positive patients vs TP53-negative patients } \\
\text { (values NR) }\end{array}$ & $<.0001$ \\
\hline Schrock 2017 [54] & $\begin{array}{l}\text { Median TMB higher in TP53-positive patients (10.1) vs TP53-negative } \\
\text { patients (5) }\end{array}$ & .001 \\
\hline
\end{tabular}

Abbreviations: NR, not reported; PD-1, programmed cell death-1; PD-L1, programmed death ligand 1; TMB, tumor mutational burden.

identified that reported TMB in lung cancer, $37(46 \%)$ reported $\mathrm{TMB}$ as mut/Mb $[16,28,30,33-35,37$, $40,41,45,46,48,54,60,61,63,66,67,69-87], 34$ $(42 \%)$ reported TMB as total mutations $[1,10,32,36$, $38,39,42-44,47,49-51,53,55-57,59,62,64,65$, 68, 88-99], and two reported both mut/MB and total mutations [31, 100]; eight publications did not report TMB in either mut/MB or total mutations [27, 29, 52, 101-105].

In total, 33 publications (41\%) classified TMB by high versus low or intermediate levels $[1,10,16$,
$28,30,32-37,39,41-44,48,51,54-56,60,63,66$, 69-71, 75, 84, 86, 91, 99, 102]. However, among these publications, various units were used to define the threshold of high or low/intermediate TMB: 16 publications (48\%) classified TMB by mut/Mb (median threshold [range]: 20 [10-20]) [16, 28, 33, 35, 37, 41, $48,54,63,66,69-71,75,84,91], 10$ publications $(30 \%)$ used a percentile to categorize TMB status [1, 30, 34, $36,44,51,56,60,86,99]$, and six publications (18\%) categorized TMB based on total mutation count (range: 4-242) [10, 32, 39, 42, 43, 55]. 
Table 4: Publications reporting data on key patient and disease characteristics and TMB

\begin{tabular}{|c|c|c|}
\hline Publication & Main findings relating to $T M B$ and patient/disease characteristic & $P$ Value \\
\hline \multicolumn{3}{|l|}{ SMOKING } \\
\hline Kadara 2017 [57] & $\begin{array}{l}\text { TMB was higher in patients with a history of smoking vs no smoking history } \\
\text { (values NR) }\end{array}$ & .002 \\
\hline Kim 2017 [58] & $\begin{array}{l}\text { TMB was higher in patients with a history of smoking ( } 101.5 \text { mutations) vs no } \\
\text { smoking history ( } 63.0 \text { mutations) }\end{array}$ & .43 \\
\hline Mahadevan 2017 [36] & $\begin{array}{l}\text { TMB was higher in patients with a history of smoking vs no smoking history } \\
\text { (values NR) }\end{array}$ & .047 \\
\hline Ono 2017 [60] & $\begin{array}{l}\text { TMB was higher in patients with a history of smoking vs no smoking history } \\
\text { (values NR) }\end{array}$ & .0001 \\
\hline Owada 2017 [51] & $\begin{array}{l}\text { TMB was higher in patients with a history of smoking vs no smoking history } \\
\text { (values NR) }\end{array}$ & $<.001$ \\
\hline Quek 2018 [61] & $\begin{array}{l}\text { TMB was higher in patients with a history of smoking }(11.6 \mathrm{mut} / \mathrm{Mb}) \text { vs no } \\
\text { smoking history }(4.0 \mathrm{mut} / \mathrm{Mb})\end{array}$ & .00016 \\
\hline Reck 2017 [62] & $\begin{array}{l}\text { TMB was higher in patients with a history of smoking ( } 199 \text { mutations) vs no } \\
\text { smoking history ( } 60 \text { mutations) }\end{array}$ & .004 \\
\hline Rizvi 2015 [1] & $\begin{array}{l}\text { TMB was higher in patients with a history of smoking vs no smoking history } \\
\text { (values NR) }\end{array}$ & .08 \\
\hline Schrock 2016 [63] & $\begin{array}{l}\text { TMB was higher in patients with a history of smoking }(10.4 \mathrm{mut} / \mathrm{Mb}) \text { vs no } \\
\text { smoking history }(3.3 \mathrm{mut} / \mathrm{Mb})\end{array}$ & NR \\
\hline Shim 2015 [64] & $\begin{array}{l}\text { TMB was higher in patients with a history of smoking vs no smoking history } \\
\text { (values NR) }\end{array}$ & $<.0001$ \\
\hline Wang 2017 [42] & $\begin{array}{l}\text { TMB was higher in patients with a history of smoking vs no smoking history } \\
\text { (values NR) }\end{array}$ & .00087 \\
\hline Xiao 2016 [43] & $\begin{array}{l}\text { TMB was higher in patients with a history of smoking ( } 3 \text { mut/Mb) vs no smoking } \\
\text { history }(2 \mathrm{mut} / \mathrm{Mb})\end{array}$ & .00139 \\
\hline Xiao 2017 [65] & $\begin{array}{l}\text { TMB was higher in patients with a history of smoking ( } 126 \text { mutations) vs no } \\
\text { smoking history ( } 46 \text { mutations) }\end{array}$ & .031 \\
\hline Chae 2018 [56] & $\begin{array}{l}\text { Patients with a history of smoking were more likely to have TMB-H than TMB-L } \\
\text { (values NR) }\end{array}$ & $<.001$ \\
\hline Davis 2017 [28] & $\begin{array}{l}\text { Smoking was associated with higher TMB mutation (TMB-H: } 18 \text { patients with } \\
\text { history of smoking vs } 1 \text { patient with no history; TMB-L: } 38 \text { patients with history } \\
\text { of smoking vs } 25 \text { patient with no history) }\end{array}$ & .001 \\
\hline Lizotte 2016 [59] & Spearman’s rho: 0.5297 & .0009 \\
\hline \multicolumn{3}{|l|}{ HISTOLOGY } \\
\hline Isaka 2017 [66] & TMB higher in patients with SCC (5.6 mut/Mb) vs adenocarcinoma (1.6) & NR \\
\hline Kojima 2017 [67] & TMB higher in patients with SCC (5.6 mut/Mb) vs adenocarcinoma (1.6) & NR \\
\hline Ono 2017 [60] & TMB higher in patients with SCC vs adenocarcinoma (no values reported) & .069 \\
\hline Owada 2017 [51] & TMB higher in patients with SCC vs adenocarcinoma (no values reported) & $<.001$ \\
\hline Schrock 2017 [54] & $\begin{array}{l}\text { TMB higher in patients with non-adenocarcinoma }(9.9 \mathrm{mut} / \mathrm{Mb}) \mathrm{vs} \\
\text { adenocarcinoma }(6.8 \mathrm{mut} / \mathrm{Mb})\end{array}$ & 176 \\
\hline \multicolumn{3}{|l|}{ SEX } \\
\hline Owada 2017 [51] & TMB higher in male patients vs female (values NR) & $<.001$ \\
\hline Xiao 2016 [43] & TMB higher in male patients ( 3 mut/Mb) vs female $(2 \mathrm{mut} / \mathrm{Mb})$ & $<.001$ \\
\hline Xiao 2017 [65] & TMB higher in male patients ( 92 mutations) vs female ( 34 mutations) & $<.001$ \\
\hline
\end{tabular}




\section{CANCER STAGE}

Choi 2017 [27]

No association of disease stage and TMB in patients with stage I-III cancer

(values NR)

Kim 2018 [58] TMB higher in patients with stage I-III cancer (104.4 mutations) vs stage IV (80.0 mutations)

Xiao 2016 [43]

No clear differences in TMB between patients with stage I (2 mutations), stage II

NR ( 2 mutations), stage III ( 2 mutations) and stage IV ( 2 mutations)

Xiao 2017 [65]

No clear differences in TMB between patients with stage I (39.5 mutations),

NR stage II (74.5 mutations), stage III (59.0 mutations) and stage IV (50.5 mutations)

\begin{tabular}{lll} 
AGE & & \\
Ono 2017 [60] & TMB higher in patients aged $\geq 70$ years vs $<70$ years (values NR) & .106 \\
Wang 2017 [42] & TMB higher in patients aged $\geq 65$ years vs $<65$ years (values NR) & .0208 \\
Xiao 2016 [43] & No difference in TMB in patients aged $\geq 65$ years (2 mutations) vs $<65$ years & .616 \\
& (2 mutations) & .897 \\
Xiao 2017 [65] & TMB higher in patients aged $\geq 65$ years (80.5 mutations) vs $<65$ years & \\
& (48 mutations) & .007 \\
Zhang 2016 [68] & Log-2 transformed effect estimates for age in patient subgroups: & .30 \\
& Adenocarcinoma TP53-positive: -0.023 & .22 \\
& Adenocarcinoma TP53-negative: 0.01 & .37 \\
\hline
\end{tabular}

Abbreviations: mut/MB, mutations per DNA megabase; NR, not reported; SCC, squamous cell carcinoma; TMB, total mutational burden.

Table 5: Summary of reporting of TMB testing practices reported by 81 included publications

\begin{tabular}{ll}
\hline Genes tested, $\boldsymbol{n}(\mathbf{\%})$ & $27(33 \%)[10,30,32-36,39-41,44,46,48,55,63,70,71,73-75,86,87,91,94$, \\
& $100,101,104]$ \\
Median (range) reported values & $315(15-592)$ \\
Mbs tested, $\boldsymbol{n}(\%)$ & $10(12 \%)[16,33,40,45,54,63,70,71,81,84]$ \\
Median (range) reported values & $1.1(0.8-1.2)$ \\
Units used for TMB & \\
measurement, $\boldsymbol{n}(\%)$ & $37(46 \%)[16,28,30,33-35,37,40,41,45,46,48,54,60,61,63,66,67,69-87]$ \\
Mut/Mb & $34(42 \%)[1,10,32,36,38,39,42-44,47,49-51,53,55-57,59,62,64,65,68$, \\
Total mutations & $88-99]$ \\
& $2(2 \%)[31,100]$ \\
Both & $8(10 \%)[27,29,52,101-105]$ \\
Other units & $33(41 \%)[1,10,16,28,30,32-37,39,41-44,48,51,54-56,60,63,66,69-71$, \\
TMB threshold defined, $\boldsymbol{n}(\%)$ & $75,84,86,91,99,102]$ \\
& $16(48 \%)[16,28,33,35,37,41,48,54,63,66,69-71,75,84,91]$ \\
By mut/Mb & $20(10-20)$ \\
Median (range) reported values & $10(30 \%)[1,30,34,36,44,51,56,60,86,99]$ \\
By percentiles & $50(50-85)$ \\
Median (range) reported values & $6(18 \%)[10,32,39,42,43,55]$ \\
By total mutations & $4-242$ \\
Range of reported values &
\end{tabular}

Abbreviations: mut/Mb, mutations per DNA megabase; TMB, tumor mutational burden. 


\section{MATERIALS AND METHODS}

A systematic literature review based on an a priori protocol (available upon request) was conducted, following Preferred Reporting Items for Systematic Reviews and Meta-Analyses (PRISMA) [22] and Cochrane Handbook for Systematic Reviews of Interventions [23] guidance.

\section{Literature search/data sources}

Search strategies combined keywords and controlled vocabulary; full search strategy details are presented in Supplementary Tables 1-3. Bibliographic databases (EMBASE, Scopus, Ovid MEDLINE ${ }^{\circledR}$ and Ovid Emcare) were searched from January 2012 until April 2018 for publications in the English language only. Methodological filters for both randomized controlled trials and observational studies were used. Both ClinicalTrials. gov and the International Clinical Trials Registry Platform were searched from January 2016 until April 2018 for ongoing, completed and in-progress studies. Conference abstracts were identified in Embase, and from review of the 20162018 conference proceedings for the American Society of Clinical Oncology (ASCO), European Society for Medical Oncology (ESMO), Society for Immunotherapy of Cancer (SITC), International Association for the Study of Lung Cancer (IASLC), and American Association for Cancer Research (AACR). Reference lists of included studies and related reviews were scanned for references missed by other methods of searching.

\section{Publication inclusion criteria}

Publications reporting controlled or observational studies (excluding case reports and case series) in adults receiving therapy for any stage or histology of lung cancer were included. Publications that evaluated TMB solely by liquid biopsy were excluded, as this method has not yet been fully validated and evidence is new and emerging [24]; however, publications reporting results from both tissue and liquid biopsies were included. Definitions of "low", "intermediate" and "high" levels of TMB were based on those used in the publications. Studies were included if they evaluated the association of TMB with clinical efficacy outcomes, biomarkers, or patient and disease characteristics. Clinical efficacy outcomes of interest included OS, PFS, ORR, DCB, DCR, and DoT. Biomarkers included PD-L1 expression, MSI status, MMR pathway gene mutations, DNA polymerase gene mutations, and any other genomic alterations. Clinical and demographic patient characteristics included sex, age, smoking status, cancer stage, and histology. Results for biomarkers and patient/disease characteristics are presented only for outcomes reported by at least three publications. Reported statistical comparisons were considered significant only when $P<.05$.

\section{Screening, study selection, and data extraction}

Screening of search results was performed using Covidence [25], and was conducted independently by two reviewers. Search results were initially screened for eligibility based on review of titles and abstracts. After initial exclusion of publications that clearly did not meet inclusion criteria based on screening review of title and abstract, the full text of each remaining publication was reviewed. Data were extracted independently by two reviewers using Covidence. Disagreements were resolved by discussion or by a third reviewer.

\section{Evaluation of study quality and publication bias}

Risk of bias was assessed using the Criteria for Critically Appraising Studies of Prognostic Tests for studies reporting clinical outcomes [26]. This method was adopted owing to the data reported and the purpose of the review. Bias was considered based on the following questions: "Was an inception cohort assembled?"; "Was the referral pattern described?"; "Were laboratory and clinical outcomes assessed in a blinded fashion?"; "Was complete follow-up achieved?"; "Was adjustment for extraneous prognostic factors carried out?"; "Were appropriate statistical methods used?" Two reviewers graded each study as low, high, or unclear risk of bias for each of the study design features, with discrepancies resolved by a third reviewer.

\section{DISCUSSION}

Despite inconsistencies in the definitions and reporting of TMB, high TMB appeared to be associated with greater clinical benefit (particularly ORR and PFS) among patients receiving immunotherapy for lung cancer. This suggests that TMB, in addition to PD-L1 expression level and dMMR/MSI-H status, may have clinical utility in identifying patients likely to respond to immunotherapies. It should be noted that almost all publications included in our clinical efficacy analysis reported exclusively on patients with NSCLC, with one assessing a cohort in which $94 \%$ of patients had NSCLC [36], and one reporting on patients with SCLC [32]. Clinical outcomes in the study assessing patients with SCLC were qualitatively similar to the broader NSCLC findings, with improved OS, PFS, and ORR in patients with high TMB. These findings are supported by recent observations from the CheckMate 227 study, the first phase 3 trial in lung cancer to assess immunotherapy specifically in patients with high TMB ( $\geq 10$ mut/Mb) [33]. That study reported that in patients receiving first-line therapy for NSCLC with high TMB, PFS was longer with nivolumab plus ipilimumab 
than with chemotherapy irrespective of PD-L1 levels (7.2 months vs 5.5 months; hazard ratio for disease progression or death [95\% CI]: 0.58 [0.41 to 0.81$]$ ). Other trials assessing the predictive utility of TMB as a marker of response to immunotherapy in lung cancer are underway (NCT02848651; NCT03668119).

Evidence for an association between PD-L1 expression and TMB was inconsistent. This is not entirely unexpected, as previous studies have reported that PD-L1 and TMB are independent predictors of response to immunotherapy in patients with NSCLC $[10,33]$. One retrospective analysis has reported that patients with both positive PD-L1 expression (defined as $\geq 1 \%$ expression) and high TMB had significantly improved ORR and PFS compared with patients who lacked one or both of these biomarkers [106]. Additional research is required to fully elucidate the clinical utility of TMB in combination with PD-L1 [107].

Our review found that TMB was consistently associated with TP53 alterations. Somatic alteration in the TP53 gene, which functions as a tumor suppressor in response to mutagenic cellular stresses, is one of the most frequent alterations in cancers, including lung cancers, although its predictive and/or prognostic role remains unclear [108]. Conversely, we found that TMB was negatively associated with EGFR mutations. In patients with EGFR-mut lung cancer, TMB has been shown to be associated with a poor response to tyrosine kinase inhibitors [109], and a meta-analysis of clinical trial data reported that patients with EGRFmut tumors demonstrate a poor therapeutic response to immunotherapies [110]. Recent work has been designed to elucidate the interaction between these biomarkers, with one meta-analysis reporting that concurrent TP53 and EGFR mutations predict poor response to tyrosine kinase inhibitors, but not to non-targeted therapies [111]. The precise nature of this interaction, and the involvement of TMB, remain unclear.

Several publications reported significant associations between TMB and multiple patient and disease characteristics, including a history of smoking, squamous cell lung carcinoma, and male sex. Of particular note, an association between smoking and high TMB levels was repeatedly demonstrated. This consistent finding is in line with previous observations that cancers related to chronic mutagenic exposures (such as from tobacco) exhibit the highest prevalence of mutations [15]. Interestingly, subgroup analyses of NSCLC patients receiving secondline nivolumab in the CheckMate 057 and 017 studies also found history of smoking to be associated with efficacy $[11,12]$. Collectively, these characteristics may help identify patients who are more likely to have high TMB and therefore to respond to immunotherapy.

Limitations of this study included the diversity of testing methods and reporting of TMB. A standardized threshold for classifying TMB levels as low, intermediate, and high does not currently exist; and in the identified publications not only were the units used to report TMB inconsistent, but even publications reporting TMB in identical units often used different threshold definitions. Furthermore, testing platforms differ, such as between commercial and institutional sites, with varying institutional guidance regarding the number of genes and/ or $\mathrm{Mb}$ to be tested, as well as the units used to report TMB findings. Additionally, although the majority of included data comes from solid tissue biopsies, some TMB data from evaluation of peripheral blood samples are also included. Future research is needed to fully evaluate the use of blood samples to assess TMB, and to compare these findings with TMB results from paired solid tumor samples. These inconsistencies impede any meaningful meta-analysis of the collated data. Other limitations of this study include the heterogeneity of lung cancer type and treatment regimens used. Consideration must also be given to the fact that our search included only a few highimpact congresses and may have overlooked relevant data reported elsewhere.

As most identified studies assessed TMB as a secondary/exploratory outcome, statistical power and reporting detail were generally insufficient. As a result, evidence of TMB as a predictive biomarker of response to immunotherapy in patients with lung cancer is lacking. Robust, adequately powered, observational and prospective clinical studies should continue to assess the association of TMB and other biomarkers with clinical outcomes of immunotherapy. We recommend that future studies assessing TMB adhere to standard reporting practices to enable comparison. The ongoing Friends of Cancer Research TMB Harmonization Project aims to provide a WES-based universal standard and to identify sources of variability once TMB scores from targeted panels have been aligned with the reference standard [112]. As further research is conducted with standardized TMB testing and reporting practices, a future metaanalysis may provide important evidence regarding the value of TMB testing in patients with lung cancer and other cancers.

In conclusion, these findings suggest that TMB may complement PD-L1 and dMMR/MSI testing in identifying patients among the lung cancer population who are likely to have good outcomes with immunotherapy. Robust, adequately powered, prospective and observational studies assessing TMB using standardized methodology are required to fully inform treatment decisions.

\section{ACKNOWLEDGMENTS}

This study was sponsored by Bristol-Myers Squibb. Medical writing assistance was provided by Martin Bell, $\mathrm{PhD}$, of Evidence Medical Affairs, and was funded by 
Bristol-Myers Squibb. The authors wish to acknowledge Komal Singh, Gabriel Krigsfeld, Signe Fransen, and Lisa Siegartel for their assistance in conducting and providing feedback on this systematic literature review. Michelle Fiander was an employee of the Department of Pharmacotherapy, University of Utah, UT at the time of initiation of the review. BMS policy on data sharing may be found at https:/www.bms.com/researchers-andpartners/independent-research/data-sharing-requestprocess.html.

\section{CONFLICTS OF INTEREST}

Beata Korytowsky, John-Michael Thomas, Florencio Calderon and Teresa M. Zyczynski are employees of, and hold stock in, Bristol-Myers Squibb Company.

\section{FUNDING}

Research Support: Funding was provided by Bristol-Myers Squibb.

Disclaimers: Extracts from this systematic review were presented as a poster at the Society for Immunotherapy of Cancer (SITC) 33rd Annual Meeting, November 7-11, 2018, Washington, DC, USA.

\section{REFERENCES}

1. Rizvi NA, Hellmann MD, Snyder A, Kvistborg P, Makarov V, Havel JJ, Lee W, Yuan J, Wong P, Ho TS, Miller ML, Rekhtman N, Moreira AL, et al. Cancer immunology. Mutational landscape determines sensitivity to PD-1 blockade in non-small cell lung cancer. Science. 2015; 348:124-28. https://doi.org/10.1126/science.aaa1348. [PubMed]

2. Snyder A, Makarov V, Merghoub T, Yuan J, Zaretsky JM, Desrichard A, Walsh LA, Postow MA, Wong P, Ho TS, Hollmann TJ, Bruggeman C, Kannan K, et al. Genetic basis for clinical response to CTLA-4 blockade in melanoma. N Engl J Med. 2014; 371:2189-99. https://doi.org/10.1056/ NEJMoa1406498. [PubMed]

3. Penault-Llorca F, Radosevic-Robin N. Tumor mutational burden in non-small cell lung cancer-the pathologist's point of view. Transl Lung Cancer Res. 2018; 7:716-21. https:// doi.org/10.21037/tlcr.2018.09.26. [PubMed]

4. Chalmers ZR, Connelly CF, Fabrizio D, Gay L, Ali SM, Ennis R, Schrock A, Campbell B, Shlien A, Chmielecki J, Huang F, He Y, Sun J, et al. Analysis of 100,000 human cancer genomes reveals the landscape of tumor mutational burden. Genome Med. 2017; 9:34. https://doi.org/10.1186/ s13073-017-0424-2. [PubMed]

5. Rosenberg JE, Hoffman-Censits J, Powles $\mathrm{T}$, van der Heijden MS, Balar AV, Necchi A, Dawson N, O’Donnell PH, Balmanoukian A, Loriot Y, Srinivas S, Retz MM, Grivas P, et al. Atezolizumab in patients with locally advanced and metastatic urothelial carcinoma who have progressed following treatment with platinum-based chemotherapy: a single-arm, multicentre, phase 2 trial. Lancet. 2016; 387:1909-20. https://doi.org/10.1016/S01406736(16)00561-4. [PubMed]

6. Lawrence MS, Stojanov P, Polak P, Kryukov GV, Cibulskis K, Sivachenko A, Carter SL, Stewart C, Mermel CH, Roberts SA, Kiezun A, Hammerman PS, McKenna A, et al. Mutational heterogeneity in cancer and the search for new cancer-associated genes. Nature. 2013; 499:214-18. https:// doi.org/10.1038/nature12213. [PubMed]

7. Schumacher TN, Schreiber RD. Neoantigens in cancer immunotherapy. Science. 2015; 348:69-74. https://doi. org/10.1126/science.aaa4971. [PubMed]

8. Yarchoan M, Hopkins A, Jaffee EM. Tumor mutational burden and response rate to PD-1 inhibition. N Engl J Med. 2017; 377:2500-01. https://doi.org/10.1056/ NEJMc1713444. [PubMed]

9. Gong J, Chehrazi-Raffle A, Reddi S, Salgia R. Development of PD-1 and PD-L1 inhibitors as a form of cancer immunotherapy: a comprehensive review of registration trials and future considerations. J Immunother Cancer. 2018; 6:8. https://doi.org/10.1186/s40425-018-0316-Z. [PubMed]

10. Carbone DP, Reck M, Paz-Ares L, Creelan B, Horn L, Steins M, Felip E, van den Heuvel MM, Ciuleanu TE, Badin F, Ready N, Hiltermann TJ, Nair S, et al, and CheckMate 026 Investigators. First-line nivolumab in stage IV or recurrent non-small-cell lung cancer. N Engl J Med. 2017; 376:2415-26. https://doi.org/10.1056/NEJMoa1613493. [PubMed]

11. Brahmer J, Reckamp KL, Baas $\mathrm{P}$, Crinò L, Eberhardt WE, Poddubskaya E, Antonia S, Pluzanski A, Vokes EE, Holgado E, Waterhouse D, Ready N, Gainor J, et al. Nivolumab versus docetaxel in advanced squamous-cell non-small-cell lung cancer. N Engl J Med. 2015; 373:12335. https://doi.org/10.1056/NEJMoa1504627. [PubMed]

12. Borghaei H, Paz-Ares L, Horn L, Spigel DR, Steins M, Ready NE, Chow LQ, Vokes EE, Felip E, Holgado E, Barlesi F, Kohlhäufl M, Arrieta O, et al. Nivolumab versus docetaxel in advanced nonsquamous non-small-cell lung cancer. N Engl J Med. 2015; 373:1627-39. https://doi. org/10.1056/NEJMoa1507643. [PubMed]

13. Garon EB, Rizvi NA, Hui R, Leighl N, Balmanoukian AS, Eder JP, Patnaik A, Aggarwal C, Gubens M, Horn L, Carcereny E, Ahn MJ, Felip E, et al, and KEYNOTE-001 Investigators. Pembrolizumab for the treatment of nonsmall-cell lung cancer. N Engl J Med. 2015; 372:2018-28. https://doi.org/10.1056/NEJMoa1501824. [PubMed]

14. Boyiadzis MM, Kirkwood JM, Marshall JL, Pritchard CC, Azad NS, Gulley JL. Significance and implications of FDA approval of pembrolizumab for biomarker-defined disease. J Immunother Cancer. 2018; 6:35. https://doi.org/10.1186/ s40425-018-0342-x. [PubMed] 
15. Alexandrov LB, Nik-Zainal S, Wedge DC, Aparicio SA, Behjati S, Biankin AV, Bignell GR, Bolli N, Borg A, Børresen-Dale AL, Boyault S, Burkhardt B, Butler AP, et al, and Australian Pancreatic Cancer Genome Initiative, and ICGC Breast Cancer Consortium, and ICGC MMML-Seq Consortium, and ICGC PedBrain. Signatures of mutational processes in human cancer. Nature. 2013; 500:415-21. https://doi.org/10.1038/nature12477. [PubMed]

16. Goodman AM, Kato S, Bazhenova L, Patel SP, Frampton GM, Miller V, Stephens PJ, Daniels GA, Kurzrock R. Tumor mutational burden as an independent predictor of response to immunotherapy in diverse cancers. Mol Cancer Ther. 2017; 16:2598-608. https://doi.org/10.1158/15357163.MCT-17-0386. [PubMed]

17. Campesato LF, Barroso-Sousa R, Jimenez L, Correa BR, Sabbaga J, Hoff PM, Reis LF, Galante PA, Camargo AA. Comprehensive cancer-gene panels can be used to estimate mutational load and predict clinical benefit to PD-1 blockade in clinical practice. Oncotarget. 2015; 6:3422127. https://doi.org/10.18632/oncotarget.5950. [PubMed]

18. Ng SB, Turner EH, Robertson PD, Flygare SD, Bigham AW, Lee C, Shaffer T, Wong M, Bhattacharjee A, Eichler EE, Bamshad M, Nickerson DA, Shendure J. Targeted capture and massively parallel sequencing of 12 human exomes. Nature. 2009; 461:272-76. https://doi.org/10.1038/ nature08250. [PubMed]

19. Frampton GM, Fichtenholtz A, Otto GA, Wang K, Downing SR, He J, Schnall-Levin M, White J, Sanford EM, An P, Sun J, Juhn F, Brennan K, et al. Development and validation of a clinical cancer genomic profiling test based on massively parallel DNA sequencing. Nat Biotechnol. 2013; 31:102331. https://doi.org/10.1038/nbt.2696. [PubMed]

20. Johnson DB, Frampton GM, Rioth MJ, Yusko E, Xu Y, Guo X, Ennis RC, Fabrizio D, Chalmers ZR, Greenbowe J, Ali SM, Balasubramanian S, Sun JX, et al. Targeted next generation sequencing identifies markers of response to PD-1 blockade. Cancer Immunol Res. 2016; 4:959-67. https://doi.org/10.1158/2326-6066.CIR-16-0143. [PubMed]

21. US FDA. FDA announces approval, CMS proposes coverage of first breakthrough-designated test to detect extensive number of cancer biomarkers. 2017. Available at: https:// www.fda.gov/NewsEvents/Newsroom/PressAnnouncements/ ucm587273.htm. Accessed April 1, 2019.

22. Moher D, Liberati A, Tetzlaff J, Altman DG, and PRISMA Group. Preferred reporting items for systematic reviews and meta-analyses: the PRISMA statement. PLoS Med. 2009; 6:e1000097. https://doi.org/10.1371/journal.pmed.1000097. [PubMed]

23. Higgins JP, Green S. Cochrane Handbook for Systematic Reviews of Interventions Version 5.1.0 [updated March 2011]. The Cochrane Collaboration. 2011.

24. Fenizia F, Pasquale R, Roma C, Bergantino F, Iannaccone A, Normanno N. Measuring tumor mutation burden in nonsmall cell lung cancer: tissue versus liquid biopsy. Transl
Lung Cancer Res. 2018; 7:668-77. https://doi.org/10.21037/ tlcr.2018.09.23. [PubMed]

25. Veritas Health Innovation. Covidence systematic review software. Melbourne, Australia. 2018. Available at https:// www.covidence.org. Accessed April 2018.

26. Levine MN, Browman GP, Gent M, Roberts R, Goodyear M. When is a prognostic factor useful? A guide for the perplexed. J Clin Oncol. 1991; 9:348-56. [PubMed]

27. Choi M, Kadara H, Zhang J, Parra ER, RodriguezCanales J, Gaffney SG, Zhao Z, Behrens C, Fujimoto J, Chow C, Kim K, Kalhor N, Moran C, et al. Mutation profiles in early-stage lung squamous cell carcinoma with clinical follow-up and correlation with markers of immune function. Ann Oncol. 2017; 28:83-89. https://doi. org/10.1093/annonc/mdw437. [PubMed]

28. Davis AA, Chae YK, Agte S, Pan A, Iams WT, Cruz MR, Mohindra N, Villaflor V, Giles FJ. Association of circulating tumor DNA (ctDNA) tumor mutational burden (TMB) with DNA repair mutations and response to anti-PD-1/ PD-L1 therapy in non-small cell lung cancer (NSCLC). J Clin Oncol. 2017; 35:11537. https://ascopubs.org/doi/ abs/10.1200/JCO.2017.35.15 suppl.11537.

29. Gettinger S, Choi J, Mani N, Datar I, Kaftan E, Goldberg S, Zelterman D, Politi K, Lifton R, Rimm D, Herbst $\mathrm{R}$, Schalper K. Predictive value of measuring somatic mutations and tumor infiltrating lymphocytes for PD-1 axis therapy in non-small cell lung cancer (NSCLC). J Thorac Oncol. 2017; 12:S430-31. https://doi.org/10.1016/j. itho.2016.11.501.

30. Kowanetz M, Zou W, Shames D, Cummings C, Rizvi N, Spira A, Frampton G, Leveque V, Flynn S, Mocci S, Shankar G, Funke R, Ballinger M, et al. OA20.01 Tumor mutation burden (TMB) is associated with improved efficacy of atezolizumab in $1 \mathrm{~L}$ and $2 \mathrm{~L}+$ NSCLC patients. $J$ Thorac Oncol. 2017; 12:S321-22. https://doi.org/10.1016/j. jtho.2016.11.343.

31. Haratani K, Hayashi H, Tanaka T, Kaneda H, Togashi Y, Sakai K, Hayashi K, Tomida S, Chiba Y, Yonesaka K, Nonagase Y, Takahama T, Tanizaki J, et al. Tumor immune microenvironment and nivolumab efficacy in EGFR mutation-positive non-small-cell lung cancer based on T790M status after disease progression during EGFRTKI treatment. Ann Oncol. 2017; 28:1532-39. https://doi. org/10.1093/annonc/mdx183. [PubMed]

32. Hellmann MD, Callahan MK, Awad MM, Calvo E, Ascierto PA, Atmaca A, Rizvi NA, Hirsch FR, Selvaggi G, Szustakowski JD, Sasson A, Golhar R, Vitazka P, et al. Tumor mutational burden and efficacy of nivolumab monotherapy and in combination with ipilimumab in smallcell lung cancer. Cancer Cell. 2018; 33:853-861.e4. https:// doi.org/10.1016/j.ccell.2018.04.001. [PubMed]

33. Hellmann MD, Ciuleanu TE, Pluzanski A, Lee JS, Otterson GA, Audigier-Valette C, Minenza E, Linardou H, Burgers S, Salman P, Borghaei H, Ramalingam SS, Brahmer J, et al. 
Nivolumab plus ipilimumab in lung cancer with a high tumor mutational burden. N Engl J Med. 2018; 378:2093104. https://doi.org/10.1056/NEJMoa1801946. [PubMed]

34. Hellmann MD, Sanchez-Vega F, La K, Rizvi H, Halpenny D, Plodkowski A, Long N, Chatila W, Jonsson P, Arbour KC, Chaft JE, Rudin CM, Kris MG, et al. Molecular determinants of response and resistance to anti-PD-(L)1 blockade in patients with NSCLC profiled with targeted next-generation sequencing (NGS). J Clin Oncol. 2017; 35:9015. https://doi.org/10.1200/ JCO.2017.35.15 supp1.9015.

35. Hu J, Li B, Zou B, Wang S, Qiu Y, Yan M, Zeng Z, Yang H, Guan Y, Zhang L, Chen W, Zhang Y, Mei L, et al. Abstract 620: associations of genomic alteration, tumor mutational burden with PD-L1 expression and response to immune checkpoint inhibitors in Chinese lung patients. Clin Res (Alex). 2018; 78:620. https://doi.org/10.1158/1538-7445. AM2018-620.

36. Mahadevan N, Adeni A, Hammerman P, Awad M, Gandhi L, Sholl L. Non-synonymous mutation burden in lung carcinoma is associated with durable clinical response to immune checkpoint blockade. J Thorac Oncol. 2017; 12:S428-29. https://doi.org/10.1016/j.jtho.2016.11.498.

37. Park W, Lopes G, Kwon D, Florou V, Chae YK, Warsch J, Ishkanian A, Jahanzeb M, Mudad R. Correlating iSEND and tumor mutation burden (TMB) with clinical outcomes of advanced non-small cell lung cancer (ANSCLC) patients on nivolumab. J Thorac Oncol. 2017; 12:S2005-06. https:// doi.org/10.1016/j.jtho.2017.09.943.

38. Patel K, Stein MK, Morris LK, Byrd KP, Weksler B, Schwartzberg LS, Fenton MJ, Vanderwalde AM, Martin MG. In silico analysis of non-synonymous SNPs (nsSNPs) and outcomes in non-small cell lung cancer (NSCLC) patients (pts) treated with immunotherapy (IT). J Clin Oncol. 2017; 35:e14563. https://doi.org/10.1200/ JCO.2017.35.15 suppl.e14563.

39. Roszik J, Haydu LE, Hess KR, Oba J, Joon AY, Siroy AE, Karpinets TV, Stingo FC, Baladandayuthapani V, Tetzlaff MT, Wargo JA, Chen K, Forget MA, et al. Novel algorithmic approach predicts tumor mutation load and correlates with immunotherapy clinical outcomes using a defined gene mutation set. BMC Med. 2016; 14:168. https:// doi.org/10.1186/s12916-016-0705-4. [PubMed]

40. Rozenblum AB, Ilouze M, Dudnik E, Dvir A, SoussanGutman L, Geva S, Peled N. Clinical impact of hybrid capture-based next-generation sequencing on changes in treatment decisions in lung cancer. J Thorac Oncol. 2017; 12:258-68. https://doi.org/10.1016/j.jtho.2016.10.021. [PubMed]

41. Singal G, Miller PG, Agarwala V, Li G, Gossai A, Albacker LA, Goldberg ME, He J, Frank S, Bourque D, Ivanov I, Fabrizio D, Caron T, et al. Analyzing biomarkers of cancer immunotherapy (CIT) response using a real-world clinicogenomic database. Ann Oncol. 2017; 28:v404-05. https:// doi.org/10.1093/annonc/mdx376.005.
42. Wang C, Xin S, Shi X, Zhao X, Wu K, Zhang L, Zhang $\mathrm{X}$. The correlation between mutation burden and disease free survival in patients with lung adenocarcinomas. J Clin Oncol. 2017; 35:8550. https://doi.org/10.1200/ JCO.2017.35.15 suppl.8550.

43. Xiao D, Pan H, Li F, Wu K, Zhang X, He J. Analysis of ultra-deep targeted sequencing reveals mutation burden is associated with gender and clinical outcome in lung adenocarcinoma. Oncotarget. 2016; 7:22857-64. https:// doi.org/10.18632/oncotarget.8213. [PubMed]

44. Yaghmour G, Pandey M, Ireland C, Patel K, Nunnery S, Powell D, Baum S, Wiedower E, Schwartzberg LS, Martin MG. Role of genomic instability in immunotherapy with checkpoint inhibitors. Anticancer Res. 2016; 36:4033-38. [PubMed]

45. Ross J, Goldberg M, Albacker L, Gay L, Agarwala V, Elvin J, Vergilio J, Suh J, Ramkissoon S, Severson E, Daniel S, Ali S, Schrock A, et al. 1138PD: immune checkpoint inhibitor (ICPI) efficacy and resistance detected by comprehensive genomic profiling (CGP) in non-small cell lung cancer (NSCLC). Ann Oncol. 2017; 28:mdx376.004. https://doi.org/10.1093/annonc/mdx376.004.

46. Chen M, Hu J, Wang L, Li G, Liu C, Wang M, Dai L, Wang W, Chirn G, Mu S, Chen L, Yao M, Wang K. The potential clinical application of comprehensive genomic profiling in targeted therapy and immunotherapy of lung cancer. $\mathbf{J}$ Thorac Oncol. 2017; 12:S2010. https://doi.org/10.1016/j. jtho.2017.09.956.

47. Dong ZY, Zhang JT, Liu SY, Su J, Zhang C, Xie Z, Zhou Q, Tu HY, Xu CR, Yan LX, Li YF, Zhong WZ, Wu YL. EGFR mutation correlates with uninflamed phenotype and weak immunogenicity, causing impaired response to PD-1 blockade in non-small cell lung cancer. OncoImmunology. 2017; 6:e1356145. https://doi.org/10.1080/21624 02X.2017.1356145. [ [PubMed]

48. Goldberg ME, Gay LM, Fabrizio D, Frampton GM, Elvin JA, Ross JS, Miller VA, Stephens PJ, Albacker LA. The interaction of PD-L1, TMB, and genomic alterations in NSCLC. Cancer Res. 2017; 77:1775. https://doi. org/10.1158/1538-7445.AM2017-1775.

49. Liu X, Lee J, Lee S, Cho J, Sun J, Zhang L, Pei Y, Chen L, Dolled-Filhart M, Emancipator K, Lunceford J, Chen R, Zhou W, et al. Abstract 4213: molecular biomarker study of programmed death receptor ligand 1 (PD-L1) in Korean patients with lung adenocarcinoma. Cancer Res. 2018; 78:4213. https://doi.org/10.1158/1538-7445.AM2018-4213.

50. Nakagomi T, Goto T, Hirotsu Y, Shikata D, Yokoyama Y, Higuchi R, Amemiya K, Okimoto K, Oyama T, Mochizuki H, Omata M. New therapeutic targets for pulmonary sarcomatoid carcinomas based on their genomic and phylogenetic profiles. Oncotarget. 2018; 9:10635-49. https://doi.org/10.18632/oncotarget.24365. [PubMed]

51. Owada Y, Muto S, Takagi H, Inoue $\mathrm{T}$, Watanabe $\mathrm{Y}$, Yamaura T, Fukuhara M, Okabe N, Matsumura Y, Hasegawa T, Ohsugi J, Tanaka D, Ito E, et al. Correlation 
between mutation burden of tumor and immunological/ clinical parameters in considering biomarkers of immune checkpoint inhibitors for non-small cell lung cancer (NSCLC). J Clin Oncol. 2017; 35:e23184. https://doi. org/10.1200/JCO.2017.35.15 suppl.e23184.

52. Rothberg BG, Das R, Jackson L, Lazowski H, Bai Y, O’Neill D, Roberts S, Rothberg J, Herbst R, Kim A, Boffa D, Rimm D, Detterbeck F, Tanoue L. The prognostic impact of EGFR, KRAS and TP53 somatic mutations in curatively resected early-stage lung adenocarcinomas. J Thorac Oncol. 2017; 12:S623-24. https://doi.org/10.1016/j. jtho.2016.11.801.

53. Schabath M, Dalvi T, Dai H, Crim A, Midha A, Shire N, Walker J, Greenawalt D, Lawrence D, Rigas J, Brody R, Potter D, Kumar N, et al. Molecular epidemiology of programmed cell death 1-ligand 1 (PD-L1) protein expression in non-small cell lung cancer. $\mathrm{J}$ Thorac Oncol. 2017; 12:S475-76. https://doi.org/10.1016/j. jtho.2016.11.566.

54. Schrock AB, Li SD, Frampton GM, Suh J, Braun E, Mehra R, Buck SC, Bufill JA, Peled N, Karim NA, Hsieh KC, Doria M, Knost J, et al. Pulmonary sarcomatoid carcinomas commonly harbor either potentially targetable genomic alterations or high tumor mutational burden as observed by comprehensive genomic profiling. J Thorac Oncol. 2017; 12:932-42. https://doi.org/10.1016/j.jtho.2017.03.005. [PubMed]

55. Senarathne W, Vranic S, Xiu J, Rose I, Gates P, Gatalica Z. Composition of the immune microenvironment differs between carcinomas metastatic to the lungs and primary lung carcinomas. Ann Diagn Pathol. 2018; 33:62-68. https://doi.org/10.1016/j.anndiagpath.2017.12.004. [PubMed]

56. Chae YK, Anker JF, Bais P, Namburi S, Giles FJ, Chuang JH. Mutations in DNA repair genes are associated with increased neo-antigen load and activated $\mathrm{T}$ cell infiltration in lung adenocarcinoma. Oncotarget. 2017; 9:7949-60. https://doi.org/10.18632/oncotarget.23742. [PubMed]

57. Kadara H, Choi M, Zhang J, Parra ER, Rodriguez-Canales J, Gaffney SG, Zhao Z, Behrens C, Fujimoto J, Chow C, Yoo Y, Kalhor N, Moran C, et al. Whole-exome sequencing and immune profiling of early-stage lung adenocarcinoma with fully annotated clinical follow-up. Ann Oncol. 2017; 28:75-82. $\quad$ https://doi.org/10.1093/annonc/mdw436. [PubMed]

58. Kim HS, Lee JH, Nam SJ, Ock CY, Moon JW, Yoo CW, Lee GK, Han JY. Association of PD-L1 expression with tumor-infiltrating immune cells and mutation burden in high-grade neuroendocrine carcinoma of the lung. J Thorac Oncol. 2018; 13:636-48. https://doi.org/10.1016/j. itho.2018.01.008. [PubMed]

59. Lizotte PH, Ivanova EV, Awad MM, Jones RE, Keogh L, Liu H, Dries R, Almonte C, Herter-Sprie GS, Santos A, Feeney NB, Paweletz CP, Kulkarni MM, et al. Multiparametric profiling of non-small-cell lung cancers reveals distinct immunophenotypes. JCI Insight. 2016; 1:e89014. https:// doi.org/10.1172/jci.insight.89014. [PubMed]

60. Ono A, Serizawa M, Omae K, Isaka M, Kojima H, Takahashi S, Nakashima K, Omori S, Wakuda K, Kenmotsu $\mathrm{H}$, Naito T, Murakami H, Urakami K, et al. Clinical factors associated with mutation burden in non-small cell lung cancer. Ann Oncol. 2017; 28:v578-79. https://doi. org/10.1093/annonc/mdx390.018.

61. Quek K, Lam V, Hillman T, Tang M, Song X, Mao X, Fujimoto J, Chow C, Eterovic K, Parra-Cuentas E, Behrens C, $\mathrm{Wu} \mathrm{X}, \mathrm{Ye} \mathrm{Y}$, et al. Mutational landscape of non-small cell lung adjacent normal [\#5363]. AACR 2018 Proceedings: Abstracts 3028-5930. Chicago: CTI Meeting Technology. 2018.

62. Reck M, Blais N, Juhasz E, Gorbunova V, Jones CM, Urban L, Orlov S, Barlesi F, Kio E, Keilholz U, Qin Q, Qian J, Nickner C, et al. Smoking history predicts sensitivity to PARP inhibitor veliparib in patients with advanced nonsmall cell lung cancer. J Thorac Oncol. 2017; 12:1098-108. https://doi.org/10.1016/j.jtho.2017.04.010. [PubMed]

63. Schrock AB, Frampton GM, Suh J, Chalmers ZR, Rosenzweig M, Erlich RL, Halmos B, Goldman J, Forde P, Leuenberger K, Peled N, Kalemkerian GP, Ross JS, et al. Characterization of 298 patients with lung cancer harboring MET exon 14 skipping alterations. J Thorac Oncol. 2016; 11:1493-502. https://doi.org/10.1016/j.jtho.2016.06.004. [PubMed]

64. Shim HS, Kenudson M, Zheng Z, Liebers M, Cha YJ, Hoang Ho Q, Onozato M, Phi Le L, Heist RS, Iafrate AJ. Unique genetic and survival characteristics of invasive mucinous adenocarcinoma of the lung. J Thorac Oncol. 2015; 10:1156-62. https://doi.org/10.1097/ JTO.0000000000000579. [PubMed]

65. Xiao D, Li F, Pan H, Liang H, Wu K, He J. Integrative analysis of genomic sequencing data reveals higher prevalence of LRP1B mutations in lung adenocarcinoma patients with COPD. Sci Rep. 2017; 7:2121. https://doi. org/10.1038/s41598-017-02405-9. [PubMed]

66. Isaka M, Serizawa M, Nagashima T, Ohnami S, Ohshima K, Urakami K, Kojima H, Takahashi S, Ono A, Sugino T, Takahashi T, Ohde Y, Kusuhara M, Yamaguchi K. Integrated genomic analysis to assess the molecular signature of Japanese patients with non-small cell lung cancer. J Thorac Oncol. 2017; 12:S2292. https://doi.org/10.1016/j. jtho.2017.09.1677.

67. Kojima H, Serizawa M, Takahashi S, Isaka M, Ono A, Nagashima T, Ohnami S, Ohshima K, Urakami K, Kusuhara M, Sugino T, Takahashi T, Yamaguchi K, Ohde Y. Genomic analysis to assess a molecular signature in Japanese patients with pulmonary high grade neuroendocrine carcinoma. J Thorac Oncol. 2017; 12:S2186. https://doi.org/10.1016/j. jtho.2017.09.1400.

68. Zhang W, Flemington EK, Zhang K. Mutant TP53 disrupts age-related accumulation patterns of somatic mutations in multiple cancer types. Cancer Genet. 2016; 209:376-80. https://doi.org/10.1016/j.cancergen.2016.07.001. [PubMed] 
69. Connelly C, Carrot-Zhang J, Stephens P, Frampton G. Abstract 1227: somatic genome alterations in cancer as compared to inferred patient ancestry. Cancer Res. 2018; 78:1227. https://doi.org/10.1158/1538-7445.AM2018-1227.

70. Dudnik E, Peled N, Wollner M, Onn A, Agbareya A, Nechushtan H, Kuznetsov T, Roisman L, Belilovski Rozenblum A, Geva S, Zer A, Bar J. BRAF mutant NSCLC: correlation with PD-L1 expression, TMB, MSI and response to ICPI and anti-BRAF therapy. J Thorac Oncol. 2017; 12:S1804-05. https://doi.org/10.1016/j.jtho.2017.09.455.

71. Elvin JA, Goldberg ME, Gay LM, Suh J, Vergilio JA, Ramkissoon SH, Ali SM, Schrock A, Fabrizio D, Frampton G, Miller V, Stephens P, Ross J. Profiling of tumor mutational burden and PD1/PDL1 immunohistochemistry (IHC) in non-small cell lung cancer. Lab Invest. 2017; 97:477A.

72. Faruki H, Mayhew GM, Serody JS, Hayes DN, Perou CM, Lai-Goldman M. Lung adenocarcinoma and squamous cell carcinoma gene expression subtypes demonstrate significant differences in tumor immune landscape. J Thorac Oncol. 2017; 12:943-53. https://doi.org/10.1016/j. itho.2017.03.010. [PubMed]

73. Gadgeel S, Johnson A, Horn L, Lovly C, Riess J, Campregher P, Stephens P, Ross J, Miller V, Cruz M, Ali S, Schrock A. Detection of novel activating FGFR rearrangements, truncations, and splice site alterations in NSCLC by comprehensive genomic profiling. J Thorac Oncol. 2017; 12:S1286. https://doi.org/10.1016/j. jtho.2016.11.1819.

74. Garofalo A, Sholl L, Reardon B, Taylor-Weiner A, AminMansour A, Miao D, Liu D, Oliver N, MacConaill L, Ducar M, Rojas-Rudilla V, Giannakis M, Ghazani A, et al. The impact of tumor profiling approaches and genomic data strategies for cancer precision medicine. Genome Med. 2016; 8:79. https://doi.org/10.1186/s13073-016-0333-9. [PubMed]

75. Gupta A, Connelly C, Frampton G, Chmielecki J, Ali S, Suh J, Schrock A, Ross J, Stephens P, Miller V. The druggable mutation landscape of lung adenocarcinoma. J Thorac Oncol. 2017; 12:S977. https://doi.org/10.1016/j. jtho.2016.11.1349.

76. Hu X, Fujimoto J, Ying L, Reuben A, Chen R, Chow C, Rodriguez-Canales J, Sun W, Hu J, Parra ER, Carmen B, $\mathrm{Wu} \mathrm{C}$, Mao $\mathrm{X}$, et al. Investigation of genomic and TCR Repertoire Evolution of AAH, AIS, MIA to invasive lung adenocarcinoma by multiregion exome and TCR Sequencing. J Thorac Oncol. 2017; 12:S2103. https://doi. org/10.1016/j.jtho.2017.09.1190.

77. Hu X, Fujimoto J, Ying L, Chen R, Estecio MR, Chow CW, Canales JR, Song X, Mao X, Scheet P, Kadara H, Parra Cuentas Cuentas ER, Behrens C, et al. Abstract 5377: tracking genomic and epigenomic evolution from preneoplastic lesions to lung adenocarcinoma by multiregion sequencing. Cancer Res. 2018; 78:5377. https:// doi.org/10.1158/1538-7445.AM2018-5377.
78. Kim Y, Hammerman PS, Kim J, Yoon JA, Lee Y, Sun JM, Wilkerson MD, Pedamallu CS, Cibulskis K, Yoo YK, Lawrence MS, Stojanov P, Carter SL, et al. Integrative and comparative genomic analysis of lung squamous cell carcinomas in East Asian patients. J Clin Oncol. 2014; 32:121-28. https://doi.org/10.1200/JCO.2013.50.8556. [PubMed]

79. Lou Y, Diao L, Cuentas ER, Denning WL, Chen L, Fan YH, Byers LA, Wang J, Papadimitrakopoulou VA, Behrens C, Rodriguez JC, Hwu P, Wistuba II, et al. Epithelial-mesenchymal transition is associated with a distinct tumor microenvironment including elevation of inflammatory signals and multiple immune checkpoints in lung adenocarcinoma. Clin Cancer Res. 2016; 22:3630 42. https://doi.org/10.1158/1078-0432.CCR-15-1434. [PubMed]

80. Negrao MV, Tang M, Jin Y, Chen Y, Hu X, Tang H, Xu H, Quek K, Zhang J, Mao X, Song X, Heymach JV, Wistuba I, et al. Abstract 213: exome sequencing of paired primary and relapsed small cell lung cancers reveals increased copy number aberration complexity to be associated with disease relapse. Cancer Res. 2018; 78:213. https://doi. org/10.1158/1538-7445.AM2018-213.

81. Ou S, Pavlick D, Stephens PJ, Ross J, Miller V, Ali S, Schrock A. Genomic analysis of non-small cell lung cancer (NSCLC) cases with focal and non-focal MET amplification. J Thorac Oncol. 2017; 12:S1778-79. https:// doi.org/10.1016/j.jtho.2017.09.400.

82. Ou SH, Schrock AB, Gowen K, Stephens PJ, Ross JS, Johnson ML, Lovly CM, Ali SM, Miller VA, Shaw AT. Association of ALK resistance mutations by EML4-ALK variant (v3 vs. non-v3) in ALK+ non-small cell lung cancer (NSCLC). J Clin Oncol. 2017; 35:9010. https://doi. org/10.1200/JCO.2017.35.15_suppl.9010.

83. Quek K, Li J, Fujimoto J, Zhang J, Chow CW, Behrens C, Zhang J, Weissferdt A, Swisher S, Lee JJ, Heymach J, Wistuba II, Futreal PA, Zhang J. Intratumor heterogeneity is associated with survival of patients with stage IA lung adenocarcinoma. J Thorac Oncol. 2017; 12:S1539. https:// doi.org/10.1016/j.jtho.2017.06.035.

84. Ross J, Cheng H, Perez-Soler R, Suh J, Pavlick D, Ali S, Schrock A, Elvin J, Vergilio JA, Ramkissoon S, Fabrizio D, Miller V, Stephens P, Gay L. Rictor amplification in non-small cell lung cancer: an emerging therapy target. J Thorac Oncol. 2017; 12:S362. https://doi.org/10.1016/j. jtho.2016.11.403.

85. Schrock A, Sharma N, Peled N, Bufill J, Srkalovic G, Spigel D, Fabrizio D, Frampton G, Connelly C, Lipka MB, Belilovski A, Lo J, Li Y, et al. Updated dataset assessing tumor mutation burden (TMB) as a biomarker for response to PD-1/PD-L1 targeted therapies in lung cancer (LC). J Thorac Oncol. 2017; 12:S422. https://doi.org/10.1016/j. jtho.2016.11.487.

86. Shou T, Wu X, Shang Y, Gao F, Wang W, Zhang Z, Wang B, Wang Y, Shi J, Yao M, Wang A, Wang K. PI3K/ 
mTOR pathway alterations may mediate PD-1/PD-L1 blockade resistance in non-small cell lung cancer. J Thorac Oncol. 2017; 12:S2301. https://doi.org/10.1016/j. jtho.2017.09.1700.

87. Song Z, Cheng G, Xu C, Wang W, Shao Y, Zhang Y. Clinicopathological characteristics of POLE mutation in patients with non-small-cell lung cancer. Lung Cancer. 2018; 118:57-61. https://doi.org/10.1016/j.lungcan.2018.02.004. [PubMed]

88. Anagnostou V, Smith KN, Forde PM, Niknafs N, Bhattacharya R, White J, Zhang T, Adleff V, Phallen J, Wali N, Hruban C, Guthrie VB, Rodgers K, et al. Evolution of neoantigen landscape during immune checkpoint blockade in non-small cell lung cancer. Cancer Discov. 2017; 7:264-76. https://doi.org/10.1158/2159-8290.CD-16-0828. [PubMed]

89. Chae Y, Rhee K, Park L, Cho A, Ko T, Chang S, Davis A, Bhave M, Cruz M, Iams W. Abstract 4608: Immunologic and clinical implications of harboring mutations in mismatch repair (MMR) genes in non-small cell lung cancer. Cancer Res. 2018; 78:4608. https://doi. org/10.1158/1538-7445.AM2018-4608.

90. Chae YR, Park LC, Cho A, Chang S, Ko T, Davis A, Bhave M, Cruz M, Iams W. Abstract 3693: Immunologic and clinical implications of polymerase $\delta$ (POLD) and Polymerase $\varepsilon$ (POLE) gene mutations in non-small cell lung cancer. Cancer Res. 2018; 78:3693. https://doi. org/10.1158/1538-7445.AM2018-3693.

91. Chen L. Predicting tumor mutational burden (TMB) and tumor neoantigen burden (TNB) of East Asian ANSCLC patients by a targeted genomic profiling. J Thorac Oncol. 2017; 12:S2006. https://doi.org/10.1016/i.jtho.2017.09.944.

92. Dong ZY, Zhang C, Li YF, Su J, Xie Z, Liu SY, Yan LX, Chen ZH, Yang XN, Lin JT, Tu HY, Yang JJ, Zhou Q, et al. Genetic and immune profiles of solid predominant lung adenocarcinoma reveal potential immunotherapeutic strategies. J Thorac Oncol. 2018; 13:85-96. https://doi. org/10.1016/j.jtho.2017.10.020. [PubMed]

93. Dong ZY, Zhong WZ, Zhang XC, Su J, Xie Z, Liu SY, Tu HY, Chen HJ, Sun YL, Zhou Q, Yang JJ, Yang XN, Lin JX, et al. Potential predictive value of TP53 and KRAS mutation status for response to PD-1 blockade immunotherapy in lung adenocarcinoma. Clin Cancer Res. 2017; 23:3012-24. https://doi.org/10.1158/1078-0432.CCR16-2554. [PubMed]

94. Kim HS, Han JY. Association of PD-L1 expression with tumor infiltrating immune cells and mutation burden in the high grade neuroendocrine carcinoma of the lung. J Clin Oncol. 2017; 35:8564. https://doi.org/10.1200/ JCO.2017.35.15 suppl.8564.

95. Nahar R, Zhai W, Zhang T, Takano A, Khng AJ, Lee YY, Liu X, Lim CH, Koh TP, Aung ZW, Lim TK, Veeravalli L, Yuan J, et al. Elucidating the genomic architecture of Asian EGFR-mutant lung adenocarcinoma through multi-region exome sequencing. Nat Commun. 2018; 9:216. https://doi. org/10.1038/s41467-017-02584-z. [PubMed]
96. Reuben A, Gittelman R, Zhang J, Wu CJ, Zhang J, Fujimoto J, Rodriguez-Canales J, Swisher S, Heymach JV, Robins H, Lee JJ, Futreal A, Wistuba II, Zhang J. TCR repertoire of lung cancer: an association with tumor molecular and clinicopathological features. J Thorac Oncol. 2017; 12:S1539. https://doi.org/10.1016/i.jtho.2017.06.033.

97. Shinmura K, Kato H, Kawanishi Y, Igarashi H, Goto M, Tao H, Inoue Y, Nakamura S, Misawa K, Mineta H, Sugimura H. Abnormal expressions of DNA glycosylase genes NEIL1, NEIL2, and NEIL3 are associated with somatic mutation loads in human cancer. Oxidative Med Cell Longevity. 2016; 2016:1546392. https://doi. org/10.1155/2016/1546392.

98. Villarroel-Espindola F, Yu X, Datar I, Mani N, Sanmamed M, Velcheti V, Syrigos K, Toki M, Zhao H, Chen L, Herbst RS, Schalper KA. Spatially resolved and quantitative analysis of VISTA/PD-1H as a novel immunotherapy target in human non-small cell lung cancer. Clin Cancer Res. 2018; 24:1562-73. https://doi.org/10.1158/1078-0432.CCR17-2542. [PubMed]

99. Zhang W, Edwards A, Flemington EK, Zhang K. Racial disparities in patient survival and tumor mutation burden, and the association between tumor mutation burden and cancer incidence rate. Sci Rep. 2017; 7:13639. https://doi. org/10.1038/s41598-017-13091-y. [PubMed]

100. Szustakowski JG, Geese WJ, Zerba K, Chang H. Abstract 5528: Evaluation of tumor mutation burden as a biomarker for immune checkpoint inhibitor efficacy: A calibration study of whole exome sequencing with FoundationOne ${ }^{\circledR}$. Cancer Res. 2018; 78:5528. https://doi.org/10.1158/15387445.AM2018-5528.

101. Chen MH, Tan KT, Cheng JH, Fang WL, Yeh YC, Yeh CN, Chang YC, Chen SJ, Hsiao M, Chao Y. PRKDC: A new candidate for checkpoint blockade immunotherapy? J Clin Oncol. 2017; 35:3022. https://doi.org/10.1200/ JCO.2017.35.15 suppl.3022.

102. Gatalica Z, Senarathne J, Vranic S. PD-L1 expression patterns in the metastatic tumors to the lung: A comparative study with the primary non-small cell lung cancer. Ann Oncol. 2017; 28:iii52. https://doi.org/10.1093/annonc/ mdx094.003.

103. Liu SY, Dong ZY, Zhong WZ, Wu SP, Xie Z, Tu HY, $\mathrm{Wu}$ YL. Dual positive PD-11 and CD8+ til represents a predominant subtype in NSCLC and correlates with augmented immunogenicity. J Thorac Oncol. 2017; 12:S237. https://doi.org/10.1016/j.jtho.2016.11.218.

104. Sato S, Nagahashi M, Goto T, Kitahara A, Koike T, Takada K, Okamoto T, Kodama K, Izutsu H, Nakada M, Maehara Y, Wakai T, Tsuchida M. Comprehensive genomic alterations identified by next-generation sequencing of lung adenocarcinoma in japanese population. J Thorac Oncol. 2017; 12:S531-32. https://doi.org/10.1016/j. jtho.2016.11.656.

105. Sharpnack M, Cho JH, Oezkan F, Koenig M, Kim I, Otterson G, Huang K, Carbone D, He K. The landscape 
of alteration of DNA integrity-related genes and their association with tumor mutation burden in non-small cell lung cancer. J Thorac Oncol. 2017; 12:S1795-96. https:// doi.org/10.1016/j.jtho.2017.09.435.

106. Hellmann MD, Nathanson T, Rizvi H, Creelan BC, SanchezVega F, Ahuja A, Ni A, Novik JB, Mangarin LM, Abu-Akeel M, Liu C, Sauter JL, Rekhtman N, et al. Genomic features of response to combination immunotherapy in patients with advanced non-small-cell lung cancer. Cancer Cell. 2018; 33:843-852.e4. https://doi.org/10.1016/j.ccell.2018.03.018. [PubMed]

107. Rizvi H, Sanchez-Vega F, La K, Chatila W, Jonsson P, Halpenny D, Plodkowski A, Long N, Sauter JL, Rekhtman N, Hollmann T, Schalper KA, Gainor JF, et al. Molecular determinants of response to anti-programmed cell death (PD)-1 and anti-programmed death-ligand 1 (PD-L1) blockade in patients with non-small-cell lung cancer profiled with targeted next-generation sequencing. J Clin Oncol. 2018; 36:633-41. https://doi.org/10.1200/ JCO.2017.75.3384. [PubMed]

108. Mogi A, Kuwano H. TP53 mutations in nonsmall cell lung cancer. J Biomed Biotechnol. 2011; 2011:583929. https:// doi.org/10.1155/2011/583929. [PubMed]

109. Offin M, Rizvi H, Tenet M, Ni A, Sanchez-Vega F, Li BT, Drilon A, Kris MG, Rudin CM, Schultz N, Arcila ME,
Ladanyi M, Riely GJ, et al. Tumor mutation burden and efficacy of EGFR-tyrosine kinase inhibitors in patients with EGFR-mutant lung cancers. Clin Cancer Res. 2019; 25:1063-69. https://doi.org/10.1158/1078-0432.CCR-181102. [PubMed]

110. Lee CK, Man J, Lord S, Links M, Gebski V, Mok T, Yang JC. Checkpoint inhibitors in metastatic egfr-mutated non-small cell lung cancer-a meta-analysis. J Thorac Oncol. 2017; 12:403-07. https://doi.org/10.1016/j. jtho.2016.10.007. [PubMed]

111. Zhang R, Tian P, Chen B, Wang T, Li W. The prognostic impact of TP53 comutation in EGFR mutant lung cancer patients: a systematic review and meta-analysis. Postgrad Med. 2019; 131:199-206. https://doi.org/10.1080/0032548 1.2019.1585690. [PubMed]

112. Friends of Cancer Research. Friends of Cancer Research announces launch of phase II TMB harmonization project. 2018. https://www.focr.org/sites/default/files/pdf/Friends $\% 20$ of $\% 20$ Cancer $\% 20$ Research $\% 20$ Announces $\% 20$ Launch $\% 20$ of $\% 20$ Phase $\% 20 I 1 \% 20 \mathrm{TMB} \% 20$ Harmonization $\% 20$ Project. pdf. Accessed November 19, 2018. 